KANAZAWA-04-16

\title{
Dihedral Families of Quarks, Leptons and Higgs Bosons
}

\author{
K. S. Babu ${ }^{1}$ and Jisuke Kubo ${ }^{2}$ \\ ${ }^{1}$ Department of Physics, Oklahoma State University, Stillwater, OK 74078, USA \\ ${ }^{2}$ Institute for Theoretical Physics, Kanazawa University, Kanazawa 920-1192, Japan
}

\begin{abstract}
We consider finite groups of small order for family symmetry. It is found that the binary dihedral group $Q_{6}$, along with the assumption that the Higgs sector is of type II, predicts mass matrix of a nearest neighbor interaction type for quarks and leptons. We present a supersymmetric model based on $Q_{6}$ with spontaneously induced CP phases. The quark sector contains 8 real parameters with one independent phase to describe the quark masses and their mixing. Predictions in the $\left|V_{u b}\right|-\bar{\eta}$, $\left|V_{u b}\right|-\sin 2 \beta\left(\phi_{1}\right)$ and $\left|V_{u b}\right|-\left|V_{t d} / V_{t s}\right|$ planes are given. The lepton sector contains also 9 parameters. A normal as well as an inverted spectrum of neutrino masses is possible, and we compute $V_{e 3}$. We find that $\left|V_{e 3}\right|^{2}>10^{-4}$ in the case of a normal spectrum, and $\left|V_{e 3}\right|^{2}>8 \times 10^{-4}$ in the case of an inverted spectrum. It is also found that $Q_{6}$ symmetry forbids all Baryon number violating terms of $d=4$, and the contributions to EDMs from the A terms vanish in this model.
\end{abstract}

PACS numbers: $11.30 . \mathrm{Hv}, 12.15 . \mathrm{Ff}, 14.60 . \mathrm{Pq}$ 


\section{INTRODUCTION}

The gauge interactions of the standard model $(\mathrm{SM})$ respect $U_{L}(3) \times U_{R}(3)$ family symmetry in both the leptonic and quark sectors. It is the Yukawa sector of the SM that breaks this family symmetry, and is responsible for the generation of the lepton and quark masses and their mixing. If no condition is imposed, there are $3 \times 3 \times 2 \times 2-8=28$ real free parameters in the quark sector alone, where 8 is the number of phases that can be absorbed into the phases of the quark fields. Of 28 only 10 parameters are physical parameters in the SM. That is, there are 18 redundant parameters in that sector. The presence of redundant parameters is not related to a symmetry in the SM. Even if they are set equal to zero at some energy scale, they will appear at different scales. These redundant parameters may become physical parameters when going beyond the SM, and, moreover, they can induce flavor changing neutral currents (FCNCs) and CP violating phenomena that are absent or strongly suppressed in the SM. Since the SM can not control the redundant parameters, the size of the new FCNCs and CP violating phases may be unacceptably large unless there is some symmetry, or one fine tunes their values. This is a flavor problem that can occur when going beyond the SM, and the most familiar case is the minimal supersymmetric standard model (MSSM) [1].

Reducing phenomenologically the number of the free parameters in the mass matrices of quarks and leptons had started already decades ago. One of the successful Ansätze for the quark mass matrices, first proposed by Weinberg |2, 3] and then extended by Fritzsch [4], is of a nearest neighbor interaction (NNI) type ${ }^{a}[6]-[16]$ :

$$
M=\left(\begin{array}{ccc}
0 & C & 0 \\
\pm C & 0 & B \\
0 & B^{\prime} & A
\end{array}\right)
$$

The complex parameters $B, B^{\prime}, C$ and $A$ for each of the up and down quark sectors can be made real by an appropriate phase rotation on the quark fields, and as a consequence, there are only 8 real free parameters with two independent phases. The Ansatz (11) can successfully reproduce the quark masses and the Cabibbo-Kobayashi-Maskawa (CKM) mixing matrix $V_{\mathrm{CKM}}$ 5. 11]. It has been also realized that the Ansatz (11) can be used in the leptonic sector, too

\footnotetext{
a See [5] for review.
} 
8, 9, 10, 15, 16]. Therefore, the Ansatz (11) is appropriate for unification, especially for the Pati-Salam type unification [17], in which the left-handed and right-handed fermion families can be separately unified.

It is known [6] that within the SM, any mass matrix for both up and down quarks can be simultaneously brought, without changing physics, to the from (11) with $\left|M_{12}\right| \neq\left|M_{21}\right|$. However, beyond the SM, this is no longer true, and to obtain (11) beyond the SM even with $\left|M_{12}\right| \neq\left|M_{21}\right|$, some principle should be required. In this paper we are motivated by a desire to derive the form (11) solely from a symmetry principle. To be definite, we assume that the responsible symmetry is (A) based on a nonabelian discrete group, and (B) only spontaneously broken. As we will find, (i) the smallest group that satisfies our assumptions is $Q_{6}$, a binary dihedral group with 12 elements, and (ii) the Higgs sector of the SM has to be so extended that the up- and down-type right-handed fermion families couple to their own $S U(2)_{L}$ Higgs doublets (type II Higgs). So, the Higgs sector of the MSSM fits the desired Higgs structure. Therefore, we are naturally led to consider a supersymmetric extension of the SM based on $Q_{6}$, as we will do in this paper. [ Frampton and Kephart [18] came to $Q_{6}$, but from different reasons ${ }^{\mathrm{b}}$.] We will also discuss other important consequences of the supersymmetric $Q_{6}$ model such as a solution to the SUSY flavor problem [42]. We find that CP can be spontaneously broken, and thanks to $Q_{6}$ a phase alignment for each A term (trilinear coupling of bosonic superpartners) with the corresponding Yukawa term occurs. Note that the misalignment of the phases appearing in the Yukawa and A terms is the origin of a large contribution to the electric dipole moments (EDM) of neutron etc [43]. We also find that $Q_{6}$ can forbid all the Baryon number violating $d=3$ and 4 operators, and allows only one $R$-parity violating operator with $d \leq 4$

After we discuss group theory on the dihedral groups $D_{N}$ and the binary dihedral groups $Q_{N}$ [18, 44] in Sect. II, we consider a supersymmetric extension of the SM based on $Q_{6}$ in Sect. III. There we discuss the quark sector, lepton sector and Higgs sector, separately. We make predictions in the $\left|V_{u b}\right|-\bar{\eta},\left|V_{u b}\right|-\sin 2 \beta\left(\phi_{1}\right)$ and $\left|V_{u b}\right|-V_{t d} / V_{t s} \mid$ planes as well as on the

\footnotetext{
$\mathrm{b}$ One of the first papers on discrete symmetries are $19,20,21,22,23,24,25,26$. Phenomenologically viable models based on nonabelian discrete flavor symmetries $A_{4}, S_{3}, D_{4}$ and $Q_{4}$, which can partly explain the flavor structure of quarks and leptons such as large neutrino mixing, have been recently constructed, respectively, in [27, 28, 29], 30, 31, 32, 33], 34, 35], and 36]. In 37, 38, 39, 40, 41], nonabelian discrete symmetries have been used to soften the SUSY flavor problem.
} 
average neutrino mass $\left\langle m_{e e}>\right.$ in neutrinoless double $\beta$ decay and the Dirac phase $\delta_{C P}$ in the neutrino mixing. In the last section, we briefly discuss the anomalies of discrete symmetries [45, 46, 47] introduced for the model, $R$ parity violating operators and the SUSY flavor problem, respectively.

\section{FINITE GROUPS $D_{N}$ AND $Q_{N}$}

\section{A. Definitions}

The group presentation for the dihedral groups $D_{N}$ is given by

$$
\left\{A_{D_{N}}, B_{D} ;\left(A_{D_{N}}\right)^{N}=B_{D}^{2}=E, B_{D}^{-1} A_{D_{N}} B_{D}=A_{D_{N}}^{-1}\right\}
$$

and

$$
\left\{A_{Q_{N}}, B_{Q} ;\left(A_{Q_{N}}\right)^{N}=E, B_{Q}^{2}=\left(A_{Q_{N}}\right)^{N / 2}, B_{Q}^{-1} A_{Q_{N}} B_{Q}=A_{Q_{N}}^{-1}\right\}
$$

for the binary dihedral group $Q_{N}$, where $E$ is the identity element. $2 N$ is the order of group (the number of the group elements). For the binary dihedral group $Q_{N}, N$ should be even starting with 4 , while $N$ for $D_{N}$ starts with 3 . The $2 N$ group elements are:

$$
\mathcal{G}=\left\{E, A,(A)^{2}, \ldots,(A)^{N-1}, B, A B,(A)^{2} B, \ldots,(A)^{N-1} B\right\}
$$

both for $D_{N}$ and $Q_{N}$. Using the property that the product $\left(A^{m} B\right)\left(A^{n} B\right)$ with $m, n=0, \ldots, N-$ 1 can always be brought to one of the elements of $\mathcal{G}$, one can easily see that they form a group. A two-dimensional representation of $A$ and $B$ is given by

$$
\begin{gathered}
A_{D_{N}}=A_{Q_{N}}=\left(\begin{array}{cc}
\cos \phi_{N} & \sin \phi_{N} \\
-\sin \phi_{N} & \cos \phi_{N}
\end{array}\right) \text { with } \phi_{N}=2 \pi / N, \\
B_{D}=\left(\begin{array}{cc}
1 & 0 \\
0 & -1
\end{array}\right) \text { for } D_{N}, B_{Q}=\left(\begin{array}{cc}
i & 0 \\
0 & -i
\end{array}\right) \text { for } Q_{N} .
\end{gathered}
$$

Note that $\operatorname{det} A_{Q_{N}}=\operatorname{det} B_{Q_{N}}=1$, implying that $Q_{N}$ is a subgroup of $S U(2)$. It follows that the dihedral group is a subgroup of $S O(3)$, which one sees if one embeds $A_{D_{N}}$ and $B_{D}$ into $3 \times 3$ matrices

$$
A_{D_{N}} \rightarrow\left(\begin{array}{ccc}
\cos \phi_{N} & \sin \phi_{N} & 0 \\
-\sin \phi_{N} & \cos \phi_{N} & 0 \\
0 & 0 & 1
\end{array}\right), B_{D} \rightarrow\left(\begin{array}{ccc}
1 & 0 & 0 \\
0 & -1 & 0 \\
0 & 0 & -1
\end{array}\right)
$$


It also follows that $D_{N}$ has only real representations, while $Q_{N}$ can have real as well as pseudoreal representations [18, 44]. However, the smallest binary dihedral group that contains both real and pseudo-real nonsinglet representations is $Q_{6}$, because $Q_{4}$ has only pseudo-real nonsinglet representations. Note that the irreducible representations (irreps) of $D_{N}$ and $Q_{N}$ are either one- or two-dimensional.

$Q_{N}$ is the "double-covering group" of $D_{N}$ in the following sense. Consider the matrices of $D_{N / 2}$, i.e., $A_{D_{N / 2}}$ and $B_{D}$, and define

$$
\tilde{A}_{Q_{N}}=A_{D_{N / 2}}, \tilde{B}_{Q}=B_{D}
$$

Note that $\tilde{A}_{Q_{N}}$ have exactly the same properties as $A_{Q_{N}}$. Therefore, the set

$$
\left\{E, \tilde{A}_{Q_{N}},\left(\tilde{A}_{Q_{N}}\right)^{2}, \ldots,\left(\tilde{A}_{Q_{N}}\right)^{N-1}, \tilde{B}_{Q}, \tilde{A}_{Q_{N}} \tilde{B}_{Q},\left(\tilde{A}_{Q_{N}}\right)^{2} \tilde{B}_{Q}, \ldots,\left(\tilde{A}_{Q_{N}}\right)^{N-1} \tilde{B}_{Q}\right\}
$$

is a set of $Q_{N}$ elements. Since however $\left(\tilde{A}_{Q_{N}}\right)^{N / 2}=\left(A_{D_{N / 2}}\right)^{N / 2}=E$ by definition, the $D_{N / 2}$ elements appear twice in (9).

\section{B. $Q_{6}$ group theory}

Before we consider a concrete model, we briefly outline the basic reasons why $Q_{6}$. Let us find out what symmetry can explain the right-upper $2 \times 2$ block of the mass matrix (1). Clearly, no abelian symmetry can explain it, whatever the Higgs structure is. In fact, that block with $M_{12}=-M_{21}=C$, is invariant under $S U(2)$. It is therefore invariant under its subgroups, too, in particular under $Q_{N}$. Similarly, one can interpret that the $2 \times 2$ block is invariant under $O(2)$. Note that $O(2)$ is not abelian $(2 \times 2$ orthogonal matrices with det $= \pm 1$ do not always commute with each other), and that $O(2)$ allows two different one-dimensional representations $\mathbf{1}$ and $\mathbf{1}^{\prime}$, where only $\mathbf{1}$ is the true singlet. The diagonal entries of the $2 \times 2$ block can be forbidden if $\mathbf{1}^{\prime}$ is assigned to the responsible Higgs. In fact, $O(2)$ may be regarded as a subgroup of $S O(3)$, and in this case the nonabelian finite subgroups are the dihedral groups, $D_{N}$. It turns out that to explain the whole structure of the mass matrix (11), we need to have real as well as pseudo-real representations.

The smallest group that contains both types of representations is $Q_{6}$, which is the doublecovering group of $S_{3} \sim D_{3}$. The irreps of $Q_{6}$ are $\mathbf{2}, \mathbf{2}^{\prime}, \mathbf{1}, \mathbf{1}^{\prime}, \mathbf{1}^{\prime \prime}, \mathbf{1}^{\prime \prime \prime}$, where the $\mathbf{2}$ is pseudo-real, while $\mathbf{2}^{\prime}$ is real. $\mathbf{1}, \mathbf{1}^{\prime}$ are real representations, while $\mathbf{1}^{\prime \prime}, \mathbf{1}^{\prime \prime \prime}$ are complex conjugate to each other. 
The group multiplication rules are given as follows $[18,44]$ :

$$
\begin{array}{rlll}
1^{\prime} \times 1^{\prime}=1, & 1^{\prime \prime} \times 1^{\prime \prime}=1^{\prime}, & 1^{\prime \prime \prime} \times 1^{\prime \prime \prime}=1^{\prime}, & 1^{\prime \prime} \times 1^{\prime \prime \prime}=1, \\
1^{\prime} \times 1^{\prime \prime \prime}=1^{\prime \prime}, & 1^{\prime} \times 1^{\prime \prime}=1^{\prime \prime \prime}, & 2 \times 1^{\prime}=2, & 2 \times 1^{\prime \prime}=2^{\prime}, \\
2 \times 1^{\prime \prime \prime}=2^{\prime}, & 2^{\prime} \times 1^{\prime}=2^{\prime}, & 2^{\prime} \times 1^{\prime \prime}=2, & 2^{\prime} \times 1^{\prime \prime \prime}=2 .
\end{array}
$$

Note that $\mathbf{1}^{\prime \prime}, \mathbf{1}^{\prime \prime \prime}$ and $\mathbf{2}$ are complex-valued. The complex conjugate representation $\mathbf{2}^{*}$ transforms the same way as $\mathbf{2}$, when $\mathbf{2}^{*}$ is identified as $\mathbf{2}^{*}=i \tau_{2} \mathbf{2}$, with $\tau_{2}$ being the second Pauli matrix. The Clebsch-Gordan coefficients for multiplying any of the irreps (which can be straightforwardly computed from the two-dimensional representation of $A_{Q_{N}}$ and $B_{Q}$ given in (5) and (6)) are given by

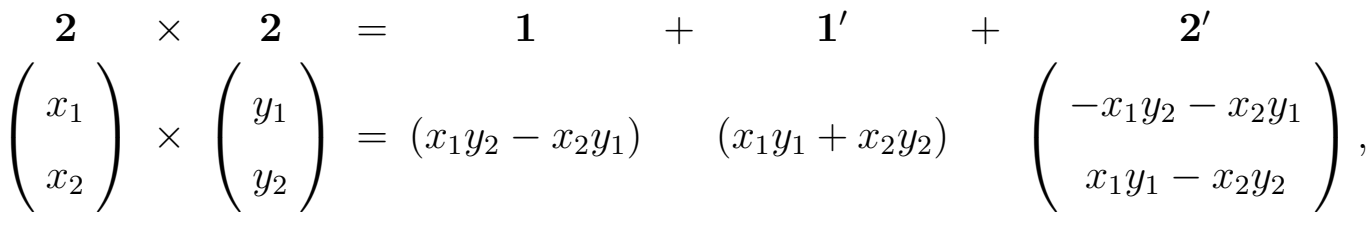

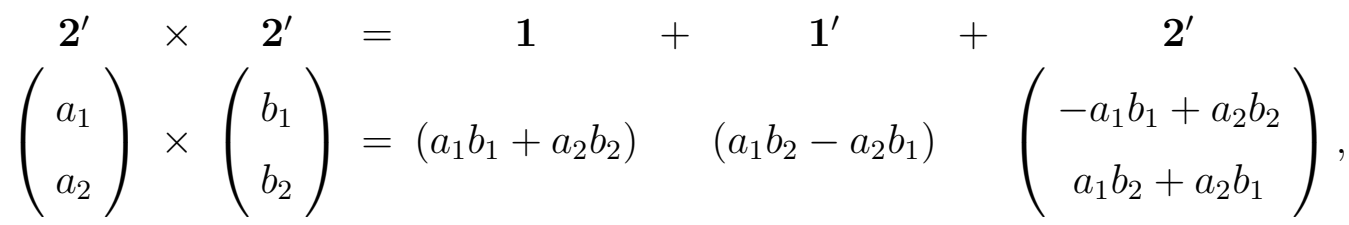

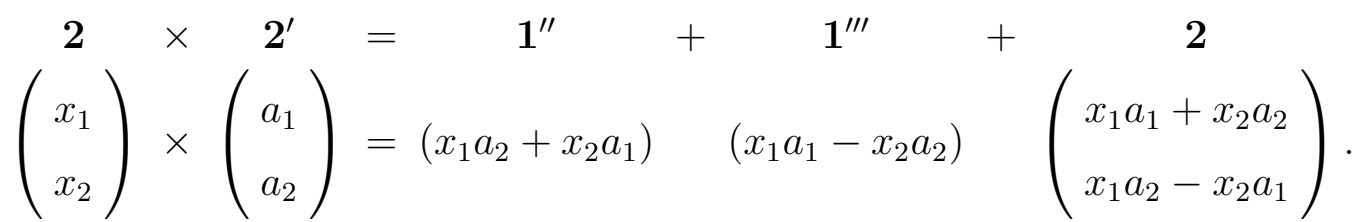

In what follows we construct a concrete model with the group theory rules given above. Multiplication rules for $D_{3,4,6,8}, Q_{4,8}$ are given in Appendix.

\section{SUPERSYMMETRIC EXTENSION OF THE STANDARD MODEL BASED ON $Q_{6}$}

We would like to derive the mass matrix (11) solely from a symmetry principle. On finds that two conditions should be met, as discussed already: (i) There should be real as well as pseudo-real nonsinglet representations, and (ii) there should be the up- and down-type Higgs $S U(2)_{L}$ doublets (type II Higgs). The smallest finite group that allows both real and pseudoreal nonsinglet representations, is $Q_{6}$, as already found out. One finds that all Higgs fields 
can not be in real representations to obtain the mass matrix (11). So, certain Higgs fields are in pseudo-real representations. If a Higgs field $H$ in a pseudo-real representation couples to a down-type fermion family, then its conjugate $H^{*}$ couples to a up-type fermion family. Since $H^{*}$ belongs to the conjugate representation, the mass matrix of the up-type family differs from that of the down-type family. Therefore, $H^{*}$ should be forbidden to couple to the up-type family in order to obtain the same form of the mass matrices for the up and down sectors. Accordingly, the Higgs sector of the MSSM fits the desired Higgs structure. Therefore, we concentrate on $Q_{6}$ and assume $N=1$ supersymmetry in the following discussions.

\section{A. $Q_{6}$ assignment}

Let us denote the fermion and Higgs fields as

$$
\begin{aligned}
\psi & =\left(\begin{array}{l}
\psi_{1} \\
\psi_{2}
\end{array}\right), \quad \psi^{c}=\left(\begin{array}{c}
-\psi_{1}^{c} \\
\psi_{2}^{c}
\end{array}\right), \quad \psi_{3}, \quad \psi_{3}^{c}, \\
H & =\left(\begin{array}{c}
H_{1} \\
H_{2}
\end{array}\right), \quad H_{3},
\end{aligned}
$$

and assume that the assignment under $Q_{6}$ is given by

$$
\psi: \mathbf{2}, \quad \psi^{c}: \mathbf{2}^{\prime}, \quad \psi_{3}: \mathbf{1}^{\prime}, \quad \psi_{3}^{c}: \mathbf{1}^{\prime \prime \prime}, \quad H: \mathbf{2}^{\prime}, \quad H_{3}: \mathbf{1}^{\prime \prime \prime}
$$

for all sectors. Here $H^{d}$ and $H_{3}^{d}$ will couple to the down quarks and charged leptons, while $H^{u}$ and $H_{3}^{u}$ will couple to the up-quark and neutrino Dirac sectors. The assignment under $Q_{6}$ is the same in all sectors. So we focus on the down-quark sector. From the Clebsch-Gordan factors given in (10) - (13), the following Yukawa couplings are found to be invariant:

$$
\mathcal{L}_{Y}=a \psi_{3} \psi_{3}^{c} H_{3}+b \psi^{T} \tau_{1} \psi_{3}^{c} H-b^{\prime} \psi_{3} \psi^{c T} i \tau_{2} H+c \psi^{T} \tau_{1} \psi^{c} H_{3}+\text { h.c. }
$$

This leads to the mass matrix

$$
M=\left(\begin{array}{ccc}
0 & c\left\langle H_{3}\right\rangle & b\left\langle H_{2}\right\rangle \\
-c\left\langle H_{3}\right\rangle & 0 & b\left\langle H_{1}\right\rangle \\
b^{\prime}\left\langle H_{2}\right\rangle & b^{\prime}\left\langle H_{1}\right\rangle & a\left\langle H_{3}\right\rangle
\end{array}\right) .
$$

If $\left\langle H_{2}\right\rangle$ vanishes, the above mass matrix has exactly the desired form (11). If $\left\langle H_{1}\right\rangle=\left\langle H_{2}\right\rangle$, we can bring the mass matrix (17) to the desired form (11) by an overall $45^{\circ}$ rotation on the 
fields of the $Q_{6}$ doublets fermions (the $\psi$ and the $\psi^{c}$ fields). So, the mass matrix (17) does not automatically lead to the desired form (11); we need to construct a Higgs sector that ensures the stability of the desired VEV structure $\left.<H_{1}\right\rangle=<H_{2}>$ (or $<H_{2}>=0$ ). A parity invariance $H_{1} \leftrightarrow H_{2}$ would ensure the VEV structure, but it is not a symmetry of (16). Therefore, the parity invariance can only be an accidental symmetry of the Higgs sector. In section III. E we will construct a Higgs sector that possesses accidentally the desired parity invariance, while the whole sector is invariant under $Q_{6} \times Z_{12 R}$. It would not work if the $Q_{6}$ doublets are replaced by doublets of $D_{3,4,5,6,7}, Q_{4}$ or $S U(2)$.

In Table 1 we write the $Q_{6}$ assignment (15) again to fix our notation, where $Z_{12 R}$ will be introduced later on when constructing the Higgs sector.

\begin{tabular}{|c|c|c|c|c|c|c|}
\hline & $Q, L$ & $U^{c}, D^{c}, E^{c}, N^{c}$ & $H^{u}, H^{d}$ & $Q_{3}, L_{3}$ & $U_{3}^{c}, D_{3}^{c}, E_{3}^{c}, N_{3}$ & $H_{3}^{u}, H_{3}^{d}$ \\
\hline$Q_{6}$ & $\mathbf{2}$ & $\mathbf{2}^{\prime}$ & $\mathbf{2}^{\prime}$ & $\mathbf{1}^{\prime}$ & $\mathbf{1}^{\prime \prime \prime}$ & $\mathbf{1}^{\prime \prime \prime}$ \\
\hline$Z_{12 R}$ & 1 & -1 & -2 & 1 & -1 & -2 \\
\hline
\end{tabular}

Table 1. $Q_{6} \times Z_{12 R}$ assignment of the matter supermultiplets. $Z_{12 R}$ will be introduced to construct a desired Higgs sector later on.

\section{B. Spontaneous CP violation}

The most stringent constraints on the soft supersymmetry breaking (SSB) parameters come from the electric dipole moments (EDM) of neutron, electron and mercury atom. Recent experiments on EDMs require that $d_{e}<64.3 \times 10^{-27}$ e cm [48], $d_{n}<6.3 \times 10^{-26}$ e cm [49] and $d_{H_{g}}<2.1 \times 10^{-28}$ e cm [50]. A misalignment of the phases appearing in the Yukawa and A terms (trilinear couplings of bosonic superpartners) is the origin of EDMs and lead to a large contribution to EDMs, so that a very fine tuning to suppress the misalignment is required [42, 43, 51, 52]. Note, however, that a flavor symmetry can not ensure the alignment of the phases. Therefore, we require that $\mathrm{CP}$ is spontaneously broken so that the origin of the $\mathrm{CP}$ phases in the Yukawa and A terms is the same. Consequently, all the coefficients appearing in the Lagrangian should be real. (Some of the couplings may be purely imaginary if the relevant fields are odd under CP.) Then tanks to $Q_{6}$ family symmetry the phases in the Yukawa and A terms after spontaneous symmetry breaking of $\mathrm{CP}$ are so aligned, that the contribution to EDMs coming from the A terms in this model vanishes. In the last section, we will discuss the 
phase alignment again when discussing the SUSY flavor problem. In the following discussions we simply assume that

$$
\left\langle H_{1}^{u}\right\rangle=\left\langle H_{2}^{u}\right\rangle,\left\langle H_{1}^{d}\right\rangle=\left\langle H_{2}^{d}\right\rangle,\left\langle H_{3}^{u}\right\rangle \neq 0,\left\langle H_{3}^{d}\right\rangle \neq 0
$$

and denote the phases of VEVs as

$$
\begin{aligned}
& \Delta \theta^{u}=\arg \left\langle H_{3}^{u}\right\rangle-\arg \left\langle H_{1}^{u}\right\rangle, \\
& \Delta \theta^{d}=\arg \left\langle H_{3}^{d}\right\rangle-\arg \left\langle H_{1}^{d}\right\rangle .
\end{aligned}
$$

In section III. E, we will construct a Higgs sector that satisfies the assumptions (18) made on VEVs. The crucial point is that the superpotential (89) posses the symmetry (92), which can ensure the desired VEV structure (18). Note that the parity invariance (92) is not imposed by hand on the superpotential (89); it is an accidentally symmetry in the Higgs sector, while the whole theory possesses $Q_{6} \times Z_{12 R}$ symmetry only.

\section{Quark sector}

$Q_{6}$ assignments of the left-handed quark supermutiplets $Q$ and $Q_{3}$, and the right-handed quark supermutiplets $U_{R}^{c}, U_{3 R}^{c}, D_{R}^{c}, D_{3 R}^{c}$ are given in Table 1. The most general $Q_{6}$ invariant superpotential for the Yukawa interactions in the quarks sector is $W_{Q}=W_{D}+W_{U}$, where

$$
W_{D}=Y_{a}^{d} Q_{3} D_{3}^{c} H_{3}^{d}+Y_{b}^{d} Q^{T} \tau_{1} D_{3}^{c} H^{d}-Y_{b^{\prime}}^{d} Q_{3} D^{c T} i \tau_{2} H^{d}+Y_{c}^{d} Q^{T} \tau_{1} D^{c} H_{3}^{d},
$$

and similarly for $W_{U}$ ( all the couplings are real). Note that neither $Z_{12 R}$ nor $R$ parity is assumed to obtain $W_{Q}$. In fact, including the lepton sector, $Q_{6}$ alone forbids all the $R$ parity violating renormalizable terms, except one term. It also forbids all the Baryon number violating Yukawa couplings. We will come to discuss this in the last section.

We assume that VEVs take the form (18), from which we obtain the mass matrix for the up and down quarks. We make an overall $45^{0}$ rotation on the $Q_{6}$ doublets, $Q, D^{c}$ and $U^{c}$, and phase rotations on the fields defined as

$$
U \rightarrow P_{u} U, U^{c} \rightarrow P_{u^{c}} U^{c}
$$


and similarly for $D$ and $D^{c}$, where

$$
\begin{aligned}
P_{u, d} & =\left(\begin{array}{ccc}
1 & 0 & 0 \\
0 \exp \left(i 2 \Delta \theta^{u, d}\right) & 0 \\
0 & 0 & \exp \left(i \Delta \theta^{u, d}\right)
\end{array}\right), \\
P_{u^{c}, d^{c}} & =\left(\begin{array}{ccc}
\exp \left(-i 2 \Delta \theta^{u, d}\right) & 0 & 0 \\
0 & 1 & 0 \\
0 & 0 \exp \left(-i \Delta \theta^{u, d}\right)
\end{array}\right) \exp \left(-i \arg \left\langle H_{3}^{u, d}\right\rangle\right) .
\end{aligned}
$$

$\left(\Delta \theta^{u, d}\right.$ are given in (19).) Then one obtains real mass matrices

$$
M_{u, d}=m_{t, b}\left(\begin{array}{ccc}
0 & q_{u, d} / y_{u, d} & 0 \\
-q_{u, d} / y_{u, d} & 0 & b_{u, d} \\
0 & b_{u, d}^{\prime} & y_{u, d}^{2}
\end{array}\right),
$$

where $m_{t}$ and $m_{b}$ are the top and bottom quark mass, respectively ${ }^{\mathrm{c}}$ We have changed our notation for the mass matrices to directly apply the result of [12] to our case. The CKM matrix $V_{\mathrm{CKM}}$ is given by

$$
V_{\mathrm{CKM}}=O_{u}^{T} P_{q} O_{d}
$$

where

$$
\begin{aligned}
O_{u}^{T} M_{u} M_{u}^{T} O_{u} & =\left(\begin{array}{ccc}
m_{u}^{2} & 0 & 0 \\
0 & m_{c}^{2} & 0 \\
0 & 0 & m_{t}^{2}
\end{array}\right), O_{d}^{T} M_{d} M_{d}^{T} O_{d}=\left(\begin{array}{ccc}
m_{d}^{2} & 0 & 0 \\
0 & m_{s}^{2} & 0 \\
0 & 0 & m_{b}^{2}
\end{array}\right), \\
P_{q} & =P_{u}^{\dagger} P_{d}=\left(\begin{array}{ccc}
1 & 0 & 0 \\
0 & \exp \left(i 2 \theta_{q}\right) & 0 \\
0 & 0 & \exp \left(i \theta_{q}\right)
\end{array}\right) \text { with } \theta_{q}=\Delta \theta^{d}-\Delta \theta^{u} .
\end{aligned}
$$

Note that there are only 8 real independent parameters, i.e., $q_{u, d}, b_{u, d}, b_{u, d}^{\prime}, y_{u, d}$, and one independent phase $\theta_{q}$ to describe $V_{\mathrm{CKM}}$ and the quark masses. Using the result of [12] we find that

\footnotetext{
${ }^{c}$ Ma 30] considered a model based on a $S_{3} \times Z_{3}$ family symmetry. In his model, the mass matrix for the down quarks is similar to $M_{d}$, but that for the up quark sector is diagonal. So tow models are distinguishable.
} 
$V_{\text {CKM }}$ can be approximately written as

$$
\begin{aligned}
& V_{u d} \simeq 1, V_{c s} \simeq \exp i 2 \theta_{q}, V_{t b} \simeq \exp i \theta_{q}, \\
& V_{u s} \simeq-y_{d} \sqrt{\frac{m_{d}}{m_{s}}}+y_{u} \sqrt{\frac{m_{u}}{m_{c}}} \exp i 2 \theta_{q}, \\
& V_{c b} \simeq \frac{y_{d}^{2}}{\sqrt{1-y_{d}^{4}}} \frac{m_{s}}{m_{b}} \exp i 2 \theta_{q}-\frac{y_{u}^{2}}{\sqrt{1-y_{u}^{4}}} \frac{m_{c}}{m_{t}} \exp i \theta_{q}, \\
& V_{t s} \simeq-\frac{y_{d}^{2}}{\sqrt{1-y_{d}^{4}}} \frac{m_{s}}{m_{b}} \exp i \theta_{q}+\frac{y_{u}^{2}}{\sqrt{1-y_{u}^{4}}} \frac{m_{c}}{m_{t}} \exp i 2 \theta_{q}, \\
& V_{u b} \simeq \frac{\sqrt{1-y_{d}^{4}}}{y_{d}} \sqrt{\frac{m_{d}}{m_{s}}} \frac{m_{s}}{m_{b}} \\
& +y_{u} \sqrt{\frac{m_{u}}{m_{c}}}\left(\frac{y_{d}^{2}}{\sqrt{1-y_{d}^{4}}} \frac{m_{s}}{m_{b}} \exp i 2 \theta_{q}-\frac{1}{y_{u}^{2} \sqrt{1-y_{u}^{4}}} \frac{m_{c}}{m_{t}} \exp i \theta_{q}\right) \text {, } \\
& V_{t d} \simeq \frac{\sqrt{1-y_{u}^{4}}}{y_{u}} \sqrt{\frac{m_{u}}{m_{c}}} \frac{m_{c}}{m_{t}} \\
& +y_{d} \sqrt{\frac{m_{d}}{m_{s}}}\left(\frac{y_{u}^{2}}{\sqrt{1-y_{u}^{4}}} \frac{m_{c}}{m_{t}} \exp i 2 \theta_{q}-\frac{1}{y_{d}^{2} \sqrt{1-y_{d}^{4}}} \frac{m_{s}}{m_{b}} \exp i \theta_{q}\right) \text {. }
\end{aligned}
$$

For the mass matrix parameters (defined in (24))

$$
\begin{aligned}
\theta_{q} & =-1.15, q_{u}=0.0002260, b_{u}=0.04596, b_{u}^{\prime}=0.08959, y_{u}=0.99746 \\
q_{d} & =0.005110, b_{d}=0.02609, b_{d}^{\prime}=0.7682, y_{d}=0.8000
\end{aligned}
$$

we obtain

$$
\begin{aligned}
m_{u} / m_{t} & =1.24 \times 10^{-5}, m_{c} / m_{t}=4.13 \times 10^{-3}, m_{d} / m_{b}=1.22 \times 10^{-3}, m_{s} / m_{b}=2.14 \times 10^{-2}, \quad(35) \\
\left|V_{u d}\right| & =0.975(1.00),\left|V_{u s}\right|=0.222(0.231),\left|V_{u b}\right|=3.64(3.59) \times 10^{-3},\left|V_{c b}\right|=4.20(3.73) \times 10^{-2}, \\
\left|V_{t d}\right| & =8.82(8.75) \times 10^{-3},\left|V_{t s}\right|=4.12(3.73) \times 10^{-2},\left|V_{t b}\right|=0.999(1.00), \\
\bar{\eta} & =0.361(0.411), \sin 2 \beta\left(\phi_{1}\right)=0.704(0.740),
\end{aligned}
$$

where $\bar{\eta}$ is one of the Wolfenstein parameters. The values in the parentheses are those obtained from the approximate formulae (28)-(33). The experimental values are [53]:

$$
\left|V_{\text {CKM }}^{\exp }\right|=\left(\begin{array}{ccc}
0.9739 \text { to } 0.9751 & 0.221 \text { to } 0.227 & 0.0029 \text { to } 0.0045 \\
0.221 \text { to } 0.227 & 0.9730 \text { to } 0.9744 & 0.039 \text { to } 0.044 \\
0.0048 \text { to } 0.014 & 0.037 \text { to } 0.043 & 0.9990 \text { to } 0.9992
\end{array}\right),
$$


and

$$
\sin 2 \beta\left(\phi_{1}\right)=0.736 \pm 0.049, \bar{\eta}=0.33 \pm 0.05 .
$$

The quark masses at $M_{Z}$ are given by [54]

$$
\begin{aligned}
m_{u} / m_{d} & =0.553 \pm 0.086(0.61), m_{s} / m_{d}=18.9 \pm 1.6(17.5) \\
m_{c} & =0.73 \pm 0.17(0.72) \mathrm{GeV}, m_{s}=0.058 \pm 0.015(0.062) \mathrm{GeV} \\
m_{t} & =175 \pm 6 \mathrm{GeV}, m_{b}=2.91 \pm 0.07 \mathrm{GeV}
\end{aligned}
$$

where the values in the parentheses are the theoretical values obtained from (35) for $m_{t}=174$ $\mathrm{GeV}$ and $m_{b}=2.9 \mathrm{GeV}$. So, we see that the 9 independent parameters can well describe 10 physical observables. Our numerical analysis show that the experimental constraints can be satisfied if

$$
-1.3<\theta_{q}<-1.0 \text { and } m_{s} / m_{d}<19
$$

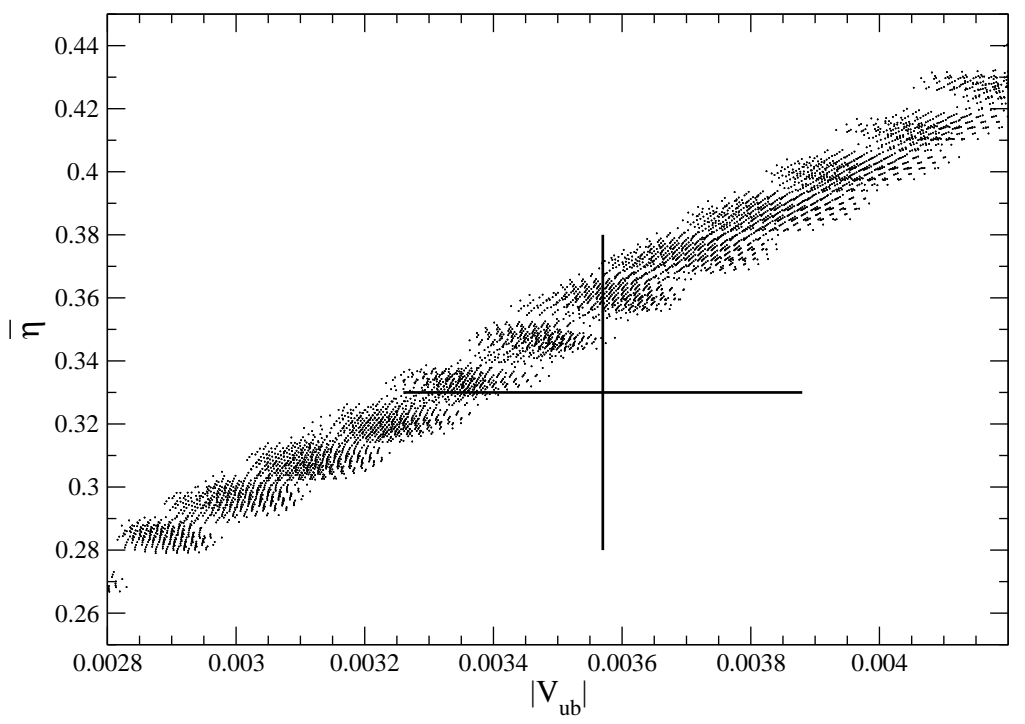

FIG. 1: Predictions in the $\left|V_{u b}\right|-\bar{\eta}$ plane. The uncertainties result from those in the quark masses (39) and in $\left|V_{u s}\right|$ and $\left|V_{c b}\right|$, where we have used $\left|V_{u s}\right|=0.2240 \pm 0.0036$ and $\left|V_{c b}\right|=(41.5 \pm 0.8) \times 10^{-3}$ 54]. The vertical and horizontal lines correspond to the experimental values $\bar{\eta}=0.33 \pm 0.05$ and $\left|V_{u b}\right|=(35.7 \pm 3.1) \times 10^{-4}[53,54]$. 


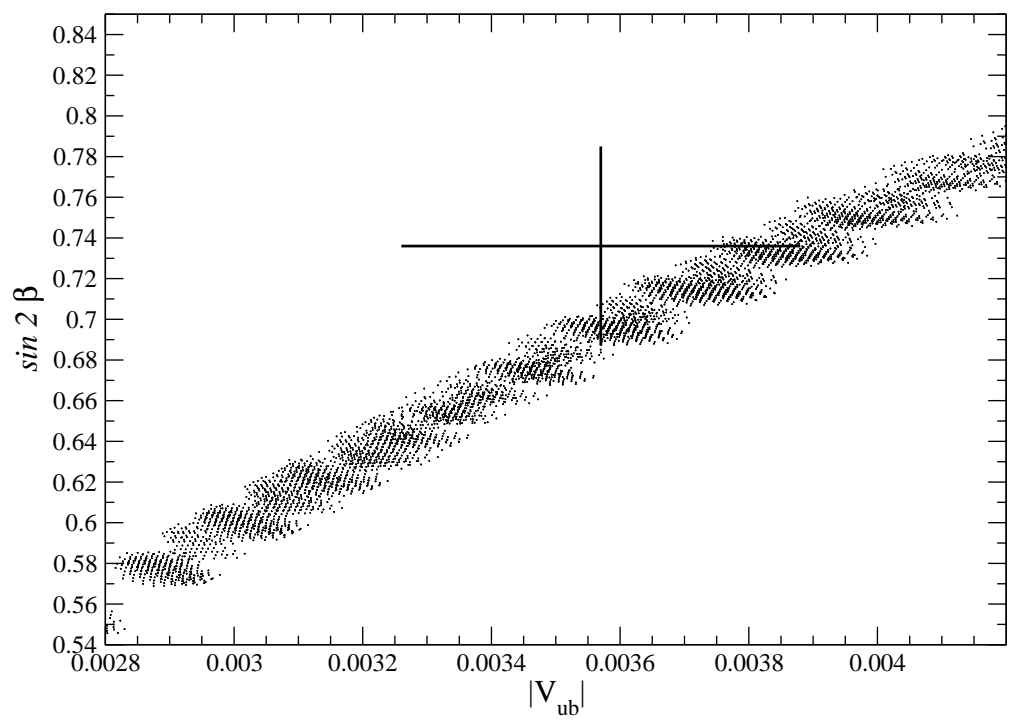

FIG. 2: Predictions in the $\left|V_{u b}\right|-\sin 2 \beta\left(\phi_{1}\right)$ plane.

In Fig. 1 we plot predicted points in the $\left|V_{u b}\right|-\bar{\eta}$ plane, where we have used the quark masses with the uncertainties given in (39) and $\left|V_{u s}\right|=0.2240 \pm 0.0036,\left|V_{c b}\right|=(41.5 \pm 0.8) \times 10^{-3}[54]$. The experimental values of $\left|V_{u b}\right|$ and $\bar{\eta}$ are indicated by the solid lines. As we see from Fig. 1 and 2 , accurate determinations of $\bar{\eta}, \sin 2 \beta\left(\phi_{1}\right)$ and $\left|V_{u b}\right|$ are crucial to test the quark sector of the model.

In Fig. 3 we plot $\left|V_{t d} / V_{t s}\right|$ against $\left|V_{u b}\right|$. From the figure we find that

$$
\left|\frac{V_{t d}}{V_{t s}}\right|=0.205-0.230 \text {. }
$$

The present experimental upper bound is 0.25 [53], which is obtained from the ratio $\Delta M_{B_{s}} / \Delta M_{B_{d}}$, where $\Delta M_{B_{d}}$ is well measured, but only an lower bound on $\Delta M_{B_{s}}$ is experimentally known. Note that a direct comparison of the theoretical values given in (41) with the experimental value is possible, only if there is no contribution to $\Delta M_{B_{s}}$ and $\Delta M_{B_{d}}$ other than those of the SM. In supersymmetric extensions of the SM and in multi-Higgs models there are extra contributions in general. Nevertheless, $\left|V_{t d} / V_{t s}\right|$ will be an important quantity for the $Q_{6}$ model. 


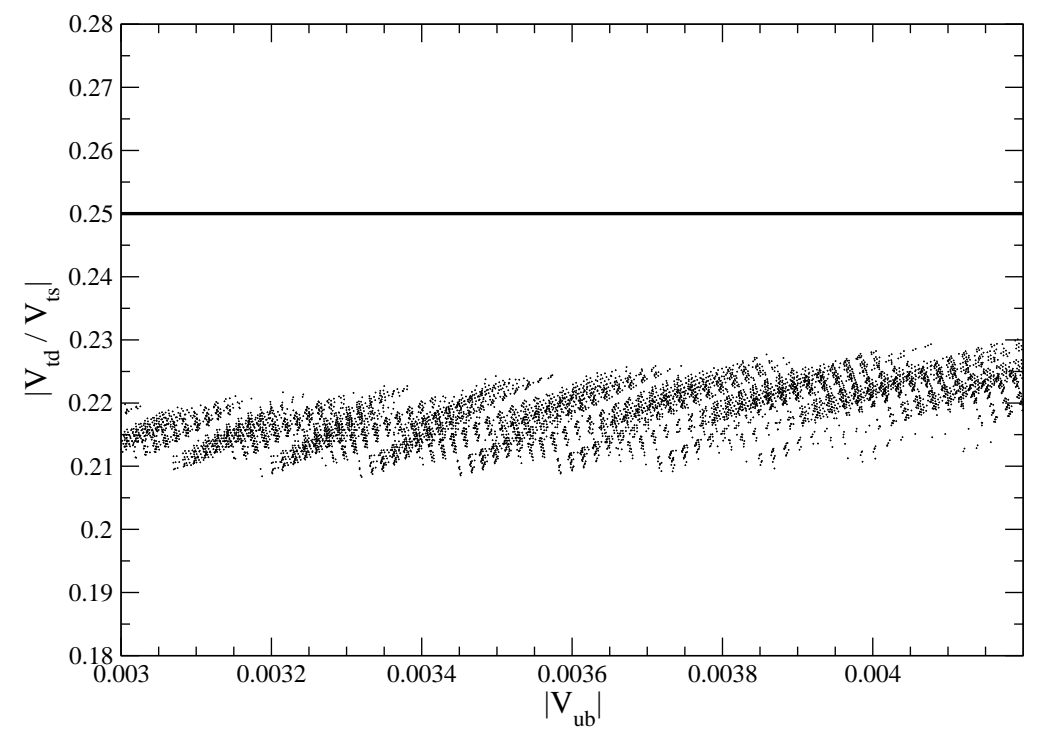

FIG. 3: Predictions in the $\left|V_{u b}\right|-\left|V_{t d} / V_{t s}\right|$ plane. The horizontal line is the experiment upper bound.

\section{Lepton sector}

As in the quark sector, the $Q_{6}$ invariant superpotential for the Yukawa interactions in the lepton sector is given by $W_{L}=W_{N}+W_{E}$, where

$$
W_{N}=Y_{a}^{\nu} L_{3} N_{3}^{c} H_{3}^{u}+Y_{b}^{\nu} L^{T} \tau_{1} N_{3}^{c} H^{u}-Y_{b^{\prime}}^{\nu} L_{3} N^{c T} i \tau_{2} H^{u}+Y_{c}^{\nu} L^{T} \tau_{1} N^{c} H_{3}^{u},
$$

and similarly for $W_{E}{ }^{\mathrm{d}}$. All the couplings are real, because we require that CP is only spontaneously broken. The charged lepton sector is very similar to the quark sector, and we find that the diagonalizing unitary matrix $U_{e}$ rotating the left-handed fermions is given by

$$
U_{e}^{\dagger}=O_{e}^{T} P_{e}^{\dagger}
$$

$\mathrm{d}$ An alterative assignment of the leptons is

$$
L: \mathbf{2}, \quad E^{c}: \mathbf{2}, \quad L_{3}: \mathbf{1}^{\prime \prime}, \quad E_{3}^{c}: \mathbf{1}^{\prime \prime}, \quad N^{c}: \mathbf{2}, \quad N_{3}^{c}: \mathbf{1},
$$

where the assignment of the quarks and Higgs supermultiplets remains unchanged. The corresponding Yukawa sector will coincide with that of the $S_{3}$ model with $Z_{2}$ in the lepton sector [31, 32]. It contains only 6 real parameters with two phases in the lepton sector and explains the maximal mixing of the atmospheric neutrinos. In that model, there exists not only Majorana phases but also a Dirac-type CP phase, which was overlooked in $\underline{31}$, 32]. Because of the spontaneous CP violation in the present case, there is only one independent phase in the lepton sector. So, the Dirac and Majorana phases are related. 
where the orthogonal matrix $O_{e}$ can be approximately written as

$$
O_{e}^{T} \simeq\left(\begin{array}{ccc}
1 & \sqrt{\frac{m_{e} \cos \phi}{m_{\mu}}} & -\sqrt{\frac{m_{e}}{m_{\mu}}} \frac{\sin \phi}{\sqrt{\cos \phi}} \\
-\sqrt{\frac{m_{e}}{m_{\mu} \cos \phi}} & \cos \phi\left(1+\frac{m_{\mu}^{2}}{m_{\tau}^{2}}\right) & -\sin \phi\left(1-\frac{m_{\mu}^{2}}{m_{\tau}^{2} \tan ^{2} \phi}\right) \\
\sqrt{\frac{m_{e}}{m_{\mu}} \frac{m_{\mu}^{2}}{m_{\tau}^{2}} \frac{\sqrt{\cos \phi}}{\sin \phi}} & \sin \phi\left(1-\frac{m_{\mu}^{2}}{m_{\tau}^{2} \tan ^{2} \phi}\right) & \cos \phi\left(1+\frac{m_{\mu}^{2}}{m_{\tau}^{2}}\right)
\end{array}\right)
$$

and

$$
P_{e}=\left(\begin{array}{ccc}
1 & 0 & \\
0 & \exp \left(i 2 \Delta \theta^{d}\right) & 0 \\
0 & 0 & \exp \left(i \Delta \theta^{d}\right)
\end{array}\right)
$$

The angel $\phi$ is an independent free parameter, and it can be assumed without loss of generality that $\sin \phi$ and $\cos \phi$ are positive.

Now let us come to the neutrino sector. Since $N^{c}$ belongs to $\mathbf{1}^{\prime \prime \prime}$ of $Q_{6}, N_{3}^{c}$ can not form a $Q_{6}$ invariant mass term, while the $Q_{6}$ doublet $N^{c}$ can do $\left(N_{1}^{c} N_{1}^{c}+N_{2}^{c} N_{2}^{c}\right)$. The absence of a mass term for $N_{3}^{c}$ would be phenomenologically inconsistent. In section III.E, when discussing the Higgs sector, we will give a way to obtain a mass term for $N_{3}^{c}$. Here we simply assume that that there is mass term for $N_{3}^{c}$. So, the mass matrix $M_{R}$ of the right-handed neutrinos is diagonal: $M_{R}=\operatorname{diag}\left(M_{1}, M_{1}, \kappa M_{3}\right)$, where $\kappa= \pm 1$ takes into account the difference of the $Q_{6}$ doublet $N^{c}$ and $Q_{6}$ singlet $N_{3}^{c}$ under CP. After an overall $45^{0}$ rotation on the $Q_{6}$ doublets, and using the sea-saw mechanism, we obtain a neutrino mass matrix. Next we make a phase rotation on the left-handed neutrinos with

$$
\begin{aligned}
& P_{\nu}=\left(\begin{array}{ccc}
\exp \left(-i r_{1}\right) & 0 & \\
0 & \exp \left(-i r_{2}\right) & 0 \\
0 & 0 & \exp \left(-i r_{3}\right)
\end{array}\right), \\
& r_{1}=r_{2}=\arg <H_{3}^{u}>, r_{3}=\arg <H_{1}^{u}>
\end{aligned}
$$

to obtain the left-handed neutrino mass matrix

$$
\begin{aligned}
M_{\nu} & =\left(\begin{array}{ccc}
c_{\nu}^{2} & 0 & c_{\nu} b_{\nu}^{\prime} \\
0 & c_{\nu}^{2}+\kappa b_{\nu}^{2} e^{-i \varphi} & \kappa a_{\nu} b_{\nu} \\
c_{\nu} b_{\nu}^{\prime} & \kappa a_{\nu} b_{\nu} & \kappa a_{\nu}^{2} e^{i \varphi}+b_{\nu}^{\prime 2}
\end{array}\right), \\
\varphi & =2\left(\arg <H_{3}^{u}>-\arg <H_{1}^{u}>\right)=2 \Delta \theta^{u} .
\end{aligned}
$$

\section{Inverted spectrum}


First we discuss the case of an inverted spectrum of neutrino masses, i.e. $m_{\nu_{2}}>m_{\nu_{1}}>m_{\nu_{3}}$. To this end, we assume that $b_{\nu}$ is small, and we treat it as perturbation:

$$
M_{\nu}=M_{\nu}^{(0)}+M_{\nu}^{(1)}+O\left(b_{\nu}^{2}\right)
$$

where

$$
M_{\nu}^{(0)}=\left(\begin{array}{ccc}
c_{\nu}^{2} & 0 & c_{\nu} b_{\nu}^{\prime} \\
0 & c_{\nu}^{2} & 0 \\
c_{\nu} b_{\nu}^{\prime} & 0 & \kappa a_{\nu}^{2} e^{i \varphi}+b_{\nu}^{\prime 2}
\end{array}\right), M_{\nu}^{(1)}=\left(\begin{array}{ccc}
0 & 0 & 0 \\
0 & 0 & \kappa a_{\nu} b_{\nu} \\
0 & \kappa a_{\nu} b_{\nu} & 0
\end{array}\right) .
$$

Because the $(1,2)$ and $(2,1)$ elements of $M_{\nu}$ is exactly zero, the correction to the eigenvalues is $O\left(b_{\nu}^{2}\right)$. But the correction to the unitary matrix $U_{\nu}$ is $O\left(b_{\nu}\right)$. This correction is found to be

$$
\begin{aligned}
U_{\nu} & =U_{\nu}^{(0)}+U_{\nu}^{(1)}+O\left(b^{2}\right), \\
U_{\nu}^{(1)} & =\left(\begin{array}{ccc}
0 & 0 & \delta U_{13} \\
\delta U_{21} & \delta U_{22} & 0 \\
0 & 0 & 0
\end{array}\right), \\
\delta U_{13} & =-\kappa a_{\nu} b_{\nu} / c b_{\nu}^{\prime}, \\
\delta U_{21} & =-\left(\cos \theta_{\nu}\right)\left(e^{-i\left(\varphi_{1}-\phi_{\nu}\right) / 2}\right) \delta U_{13}, \\
\delta U_{22} & =\left(\sin \theta_{\nu}\right)\left(e^{-i\left(\varphi_{2}-\phi_{\nu}\right) / 2}\right) \delta U_{13},
\end{aligned}
$$

where $U_{\nu}^{(0)}, \varphi_{1,2}, \phi_{\nu}$ and $\theta_{\nu}$ are given below. The unitary matrix $U_{\nu}^{(0)}$ can be obtained from

$$
U_{\nu}^{(0) T} M_{\nu}^{(0)} U_{\nu}^{(0)}=\left(\begin{array}{ccc}
m_{\nu_{1}} & 0 & 0 \\
0 & m_{\nu_{2}} & 0 \\
0 & 0 & m_{\nu_{3}}
\end{array}\right)
$$

where $m_{\nu_{i}}$ are real and positive, and

$$
U_{\nu}^{(0)}=\left(\begin{array}{ccc}
c_{\nu} e^{-i\left(\varphi_{1}-\phi_{\nu}\right) / 2} & -s_{\nu} e^{-i\left(\varphi_{2}-\phi_{\nu}\right) / 2} & 0 \\
0 & 0 & 1 \\
s_{\nu} e^{-i\left(\varphi_{1}+\phi_{\nu}\right) / 2} & c_{\nu} e^{-i\left(\varphi_{2}+\phi_{\nu}\right) / 2} & 0
\end{array}\right)
$$

with $c_{\nu}=\cos \theta_{\nu}$ etc. Note that we obtain an inverted spectrum

$$
m_{\nu_{1}}, m_{\nu_{2}}>m_{\nu_{3}}
$$


as long as $b_{\nu}$ is small. The phases and the masses satisfy to $O\left(b_{\nu}\right)$ the following relations:

$$
\begin{aligned}
m_{\nu_{3}} \sin \phi_{\nu} & =m_{\nu_{2}} \sin \varphi_{2}=m_{\nu_{1}} \sin \varphi_{1} \\
\varphi & =2 \Delta \theta^{u}=\varphi_{1}+\varphi_{2}-\frac{1}{2}(1-\kappa) \pi+(\bmod 2 \pi) \\
& \simeq \frac{1}{2}(1+\kappa) \pi+(\bmod 2 \pi) \\
m_{\nu_{3}} & =c_{\nu}^{2}, \frac{m_{\nu_{1}} m_{\nu_{2}}}{m_{\nu_{3}}}=a_{\nu}^{2} \\
\tan \phi_{\nu} & =-\frac{\kappa a_{\nu}^{2} \sin \varphi}{c_{\nu}^{2}+b_{\nu}^{2}+\kappa a_{\nu}^{2} \cos \varphi} \\
\tan ^{2} \theta_{\nu} & =\frac{\left(m_{\nu_{1}}^{2}-m_{\nu_{3}}^{2} \sin ^{2} \phi_{\nu}\right)^{1 / 2}-m_{\nu_{3}}\left|\cos \phi_{\nu}\right|}{\left(m_{\nu_{2}}^{2}-m_{\nu_{3}}^{2} \sin ^{2} \phi_{\nu}\right)^{1 / 2}+m_{\nu_{3}}\left|\cos \phi_{\nu}\right|}
\end{aligned}
$$

The last equation (61) can be rewritten as

$$
\begin{aligned}
\frac{m_{\nu_{1}}^{2}}{\Delta m_{23}^{2}-\Delta m_{21}^{2}} & =\frac{\left(1+2 t_{\nu}^{2}+t_{\nu}^{4}+r t_{\nu}^{4}\right)^{2}}{4 t_{\nu}^{2}\left(1+t_{\nu}^{2}\right)\left(1+t_{\nu}^{2}+r t_{\nu}^{2}\right) \cos ^{2} \phi_{\nu}}-\tan ^{2} \phi_{\nu} \\
& \simeq \frac{1}{\sin ^{2} 2 \theta_{12} \cos ^{2} \phi_{\nu}}-\tan ^{2} \phi_{\nu} \text { for }|r|<<1
\end{aligned}
$$

where $t_{\nu}=\tan \theta_{\nu}$ and $r=\Delta m_{21}^{2} /\left(\Delta m_{23}^{2}-\Delta m_{21}^{2}\right)$.

The mixing matrix is obtained from $O_{e}^{T} P_{e}^{\dagger} P_{\nu} U_{\nu}$, which can be brought into the form

$$
V_{\mathrm{MNS}}=\hat{V}_{\mathrm{MNS}} \times\left(\begin{array}{ccc}
1 & 0 & 0 \\
0 & e^{i \alpha} & 0 \\
0 & 0 & e^{i \beta}
\end{array}\right)
$$

where $(1,1),(1,2),(2,3)$ and $(3,3)$ elements of $\hat{V}_{\mathrm{MNS}}$ are real. $O_{e}^{T}, P_{e}, P_{\nu}$ and $U_{\nu}$ are given in (44), (45), (47) and (52). To $O\left(b_{\nu}\right)$, the mixing matrix $\hat{V}_{\text {MNS }}$ and the Majorana phases are 
approximately given by

$$
\begin{aligned}
\alpha & =\frac{1}{2}\left(\varphi_{1}-\varphi_{2}\right) \\
\beta & =-\Delta \theta^{u}-\Delta \theta^{d}+\frac{1}{2}\left(\varphi_{1}+\phi_{\nu}\right) \\
\hat{V}_{e 2} & \simeq-\sin \theta_{12} \simeq-\sin \theta_{\nu}, \\
\hat{V}_{\mu 3} & \simeq \cos \theta_{23} \simeq \cos \phi\left(1+m_{\mu}^{2} / m_{\tau}^{2}\right), \\
\hat{V}_{\tau 3} & \simeq \sin \theta_{23} \simeq \sin \phi\left(1-m_{\mu}^{2} / m_{\tau}^{2} \tan ^{2} \phi\right), \\
\hat{V}_{e 3} & =-\sin \theta_{13} e^{-i \delta_{C P}} \\
& \simeq-\kappa\left(a_{\nu} b_{\nu} / c_{\nu} b_{\nu}^{\prime}\right) e^{-i \delta_{1 C P}}+\left(m_{e} \cos \phi / m_{\mu}\right)^{1 / 2} e^{-i \delta_{2 C P}}, \\
\delta_{1 C P} & =\phi_{\nu}-\Delta \theta^{u}-\Delta \theta^{d}, \\
\delta_{2 C P} & =\phi_{\nu}-\Delta \theta^{u}+\Delta \theta^{d},
\end{aligned}
$$

where $\phi$ is defined in (44), and $\phi_{\nu}$ and $\theta_{\nu}$ are defined in (55). Since $\varphi=2 \Delta \theta^{u}$ (see (49)) and because of (57) and (60), only $\Delta \theta^{u}$ and $\Delta \theta^{d}$ are independent phases. Note that $\varphi_{2}+\varphi_{1} \simeq \pi$ and $\varphi_{2} \sim \pi$. If $\varphi_{1} \sim \pi$, we obtain $\tan \theta_{\nu}>1$.

Although $b_{\nu}$ is a free parameter, it is possible to give a minimum for $\left|V_{e 3}\right|$, because the difference of the phases $\delta_{1 C P}$ and $\delta_{2 C P}$ depends only on $\Delta \theta^{d}$ :

$$
\begin{aligned}
\left|V_{e 3}\right| & =\left|\hat{V}_{e 3}\right| \simeq\left|\sin \theta_{13}\right|>\left(m_{e} \cos \theta_{23} / m_{\mu}\right)^{1 / 2}\left|\sin 2 \Delta \theta^{d}\right| \\
& >0.057\left|\sin 2 \Delta \theta^{d}\right| \sim 0.03
\end{aligned}
$$

where we have used (67), (171) and (72), $-1.3<\theta_{q}=\Delta \theta^{d}-\Delta \theta^{u}<-1.0$ (see (401)) and $2 \Delta \theta^{u} \simeq \pi$ or $0($ see (58) $)$.

Using (57) one can express to $O\left(b_{\nu}\right) \sin 2 \alpha$ as

$$
\begin{aligned}
\sin 2 \alpha & =\sin \left(\varphi_{1}-\varphi_{2}\right) \\
& =-\frac{m_{\nu_{3}} \sin \phi_{\nu}}{m_{\nu_{1}} m_{\nu_{2}}}\left(\sqrt{m_{\nu_{2}}^{2}-m_{\nu_{3}}^{2} \sin ^{2} \phi_{\nu}}+\sqrt{m_{\nu_{1}}^{2}-m_{\nu_{3}}^{2} \sin ^{2} \phi_{\nu}}\right),
\end{aligned}
$$




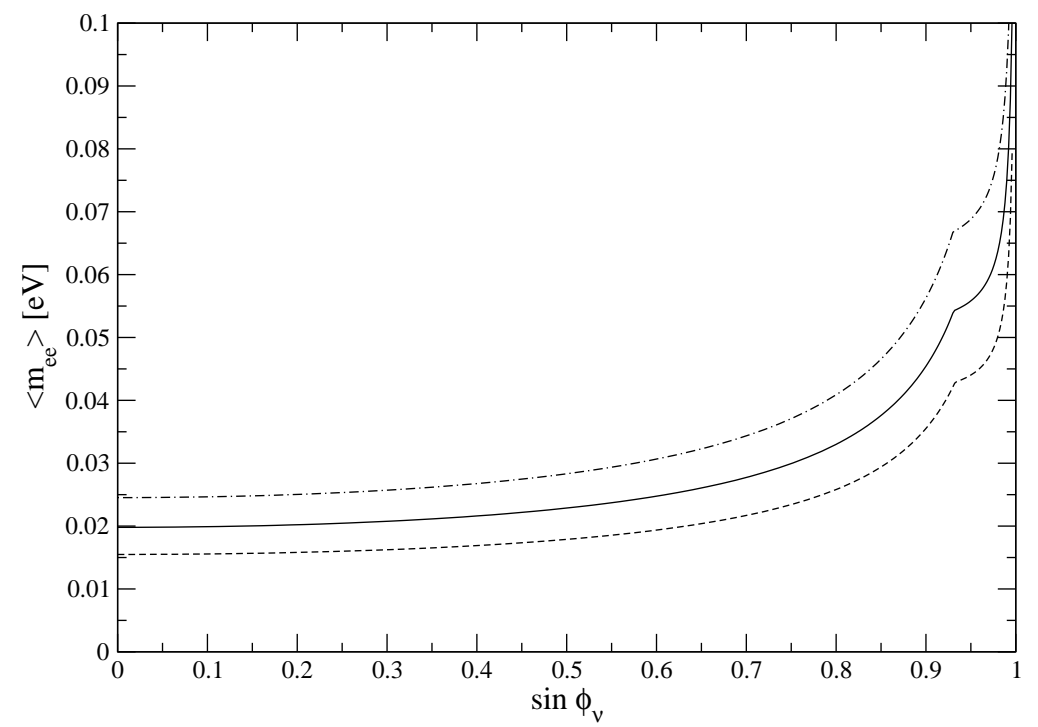

FIG. 4: The average neutrino mass $<m_{e e}>$ in the limit $b_{\nu} \rightarrow 0$ as a function of $\sin \phi_{\nu}$ with $\sin ^{2} \theta_{12}=$ 0.3 and $\Delta m_{21}^{2}=8.1 \times \mathrm{eV}^{2}$. The dashed, solid and dot-dashed lines correspond to $\Delta m_{23}^{2}=1.4,2.2$ and $3.3 \times 10^{-3} \mathrm{eV}^{2}$, respectively. The $\Delta m_{21}^{2}$ dependence is very small. The neutrino spectrum is inverted.

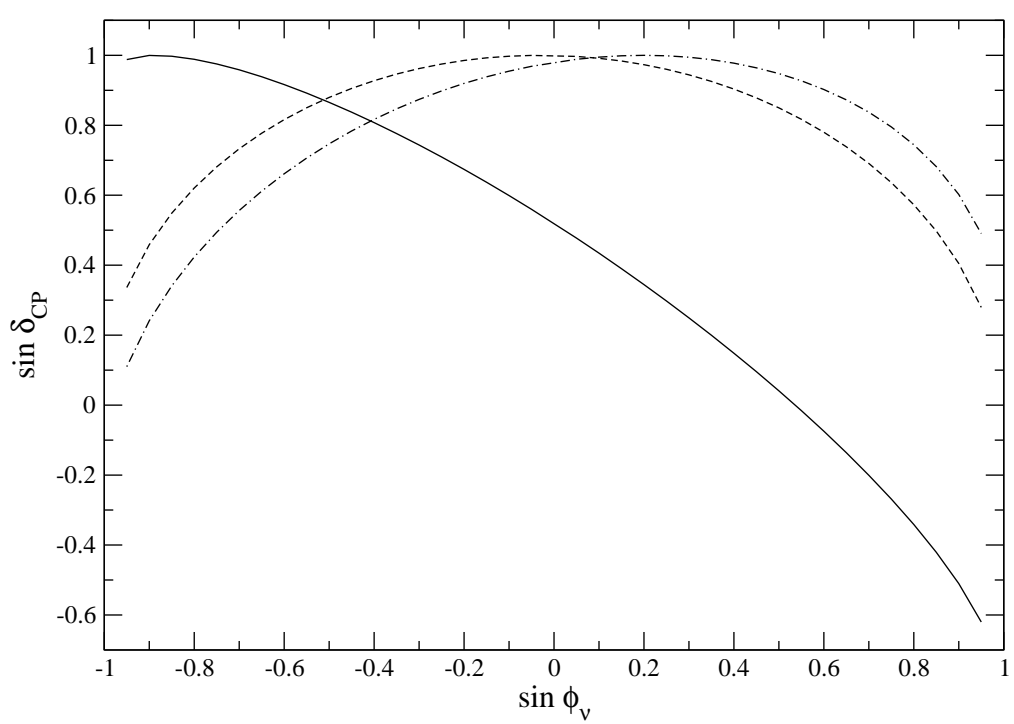

FIG. 5: $\sin \delta_{C P}$ in the limit $b_{\nu} \rightarrow 0$ versus $\sin \phi_{\nu}$ for different values of $\sin \theta_{13}$, where we use $\sin ^{2} \theta_{12}=$ $0.3, \theta_{q}=-1.15$. The solid line corresponds to $\sin \theta_{13}=0.0438$, which is the minimum for $\theta_{q}=-1.15$. The other lines are those for $\sin \theta_{13}=0.10$ (dotted), 0.20 (dot-dashed), respectively. $\theta_{q}$ is defined in (48). The result does not depend on $\kappa$.

where we have used $\varphi_{2}+\varphi_{1} \simeq \pi$ and $\varphi_{2} \sim \pi$. Eq. (60) can be rewritten in terms of measurable 
quantities:

$$
\begin{aligned}
\kappa \sin \varphi & =\kappa \sin 2 \Delta \theta^{u}=\frac{m_{\nu_{3}}}{m_{\nu_{1}}} \sin \phi_{\nu}\left[1-\left(\frac{m_{\nu_{3}}}{m_{\nu_{2}}} \sin \phi_{\nu}\right)^{2}\right]-\frac{m_{\nu_{3}}}{m_{\nu_{2}}} \sin \phi_{\nu}\left[1-\left(\frac{m_{\nu_{3}}}{m_{\nu_{1}}} \sin \phi_{\nu}\right)^{2}\right] \\
& \simeq \frac{m_{\nu_{3}}}{2 m_{\nu_{2}}^{3}} \frac{\Delta m_{21}^{2} \sin \phi_{\nu}}{\left(1-\left(m_{\nu_{3}} / m_{\nu_{2}}\right)^{2} \sin ^{2} \phi_{\nu}\right)^{1 / 2}},
\end{aligned}
$$

which relates $\phi_{\nu}$ with $\Delta \theta^{u}$. Although $\sin \varphi \simeq 0$ because of (58), $\sin \phi_{\nu}$ is not small and can vary from -1 to +1 . So, it may be better to regard $\sin \phi_{\nu}$ as independent instead of $\Delta \theta^{u}$.

The average neutrino mass $\left\langle m_{e e}\right\rangle$ can be then predicted from

$$
<m_{e e}>=\left|\sum_{i=1}^{3} m_{\nu_{i}} V_{e i}^{2}\right| \simeq\left|m_{\nu_{1}} c_{\nu}^{2}+m_{\nu_{2}} s_{\nu}^{2} \exp i 2 \alpha\right| .
$$

To obtain numerical values of $\left\langle m_{e e}\right\rangle$, we use the experimental values [55]

$$
\begin{gathered}
7.2 \times 10^{-5} \mathrm{eV}^{2} \leq \Delta m_{21}^{2} \leq 9.1 \times 10^{-5} \mathrm{eV}^{2}, 1.4 \times 10^{-3} \mathrm{eV}^{2} \leq \Delta m_{23}^{2} \leq 3.3 \times 10^{-3} \mathrm{eV}^{2} \\
0.23 \leq \sin ^{2} \theta_{12} \leq 0.38,0.34 \leq \sin ^{2} \theta_{23} \leq 0.68,\left|\sin ^{2} \theta_{13}\right|<0.047
\end{gathered}
$$

Note that $\alpha$ is given in (75) and the absolute scale of the neutrino mass can be obtained from (62). In Fig. 4 we plot $<m_{e e}>$ as a function of $\sin \phi_{\nu}$ for $\sin ^{2} \theta_{12}=0.3, \Delta m_{21}^{2}=8.1 \times 10^{-5} \mathrm{eV}^{2}$ and $\Delta m_{23}^{2}=1.4,2.2,3.3 \times 10^{-3} \mathrm{eV}^{2}$. As we can see from Fig. 2, the effective Majorana mass stays at about its minimal value $<m_{e e}>_{\min }$ for a wide range of $\sin \phi_{\nu}$. Since $<m_{e e}>_{\min }$ is approximately equal to $<m_{e e}>_{\min } \simeq \sqrt{\Delta m_{23}^{2}} / \tan 2 \theta_{12}=(0.010-0.037) \mathrm{eV}$, it is consistent with recent experiments [56, 57].

To make the case of an inverted spectrum of the model more transparent, we summarize the result in this sector. A set of the independent quantities are: three charged fermion masses, $\theta_{13}, \theta_{12}\left(\simeq \theta_{\nu}\right), \theta_{23}(\simeq \phi$ defined in (44) $), \Delta m_{21}^{2}, \Delta m_{23}^{2}$ and $\phi_{\nu}$, where $\theta_{q}=\Delta \theta^{d}-\Delta \theta^{u}$ can be determined in the quark sector, and $\Delta \theta^{u}$ is a function of $\phi_{\nu}$ given in (76). Therefore, the absolute scale for neutrino masses can be computed from (62). One of the absolute scales is the effective neutrino mass $<m_{e e}>$ in neutrinoless double $\beta$ decay, which is shown in Fig. 4. Another prediction is the Dirac phase $\delta_{C P}$ defined in (69), which is plotted as a function of $\phi_{\nu}$ for different values of $\sin \theta_{13}$ in Fig. 5 , where we have assumed $\cos \theta_{23}=1 / \sqrt{2}$. As we can see from these figures, precise measurements of the physical parameters such as $\sin \theta_{13}, \delta_{C P}$ and $<m_{e e}>$ in the neutrino sector can in principle confirm the predictions of the model.

\section{Normal spectrum}


A normal hierarchal spectrum of neutrino masses with small $\left|V_{e 3}\right|$ can be obtained if the mass parameters of $M_{\nu}$ (48) satisfy the following conditions:

$$
c_{\nu}^{2}, b_{\nu}^{\prime 2}<<b_{\nu}^{2}, a_{\nu}^{2} \text { and } \varphi=2 \Delta \theta^{u} \simeq 0
$$

So, in the lowest order approximation, we may neglect $\mathrm{CP}$ violating phases involved in the unitary matrix $U_{\nu}$. Under the conditions (80), we find that the unitary matrix $U_{\nu}$ can be approximately written as

$$
U_{\nu}^{(0)}=\left(\begin{array}{ccc}
1 & 0 & 0 \\
0 & c_{a} & s_{a} \\
0 & -s_{a} & c_{a}
\end{array}\right) \times\left(\begin{array}{ccc}
c_{\nu} & -s_{\nu} & \epsilon \\
s_{\nu} & c_{\nu} & 0 \\
\epsilon c_{\nu} & \epsilon s_{\nu} & 1
\end{array}\right)
$$

where $s_{\nu}=\sin \theta_{\nu}, s_{a}=\sin \theta_{a}$ etc, and

$$
\begin{aligned}
\epsilon & \simeq \frac{R_{\nu}}{2} \cot \theta_{a} \sin 2 \theta_{\nu} \\
R_{\nu} & =\frac{\left(m_{\nu_{2}}-m_{\nu_{1}}\right)}{m_{\nu_{3}}-m_{\nu_{2}} \sin ^{2} \theta_{\nu}-m_{\nu_{1}} \cos ^{2} \theta_{\nu}} .
\end{aligned}
$$

The mixing matrix $V_{\mathrm{MNS}}$ is obtained from $O_{e}^{T} P_{e}^{\dagger} P_{\nu} U_{\nu}$, where $O_{e}^{T}, P_{e}$ and $P_{\nu}$ are given in (44), (45), (47). Note, however, that because of the condition (80), arg $\left\langle H_{1}^{u}>\simeq \arg \left\langle H_{3}^{u}\right\rangle\right.$ so that $P_{\nu} \propto \mathbf{1}$. We find that the mixing matrix $V_{\mathrm{MNS}}$ is approximately given by

$$
\begin{aligned}
& V_{e 2} \simeq-\sin \theta_{\nu} \\
& V_{e 3} \simeq \epsilon+\sqrt{\frac{m_{e}}{m_{\mu}}}\left(\sqrt{\cos \phi} \sin \theta_{a} e^{-i 2 \Delta \theta^{d}}-\frac{\sin \phi}{\sqrt{\cos \phi}} \cos \theta_{a} e^{-i \Delta \theta^{d}}\right), \\
& V_{\mu 3} \simeq \sin \theta_{a} \cos \phi e^{-2 i \Delta \theta^{d}}-\cos \theta_{a} \sin \phi e^{-i \Delta \theta^{d}}, \\
& V_{\tau 3} \simeq \sin \theta_{a} \sin \phi e^{-2 i \Delta \theta^{d}}+\cos \theta_{a} \cos \phi e^{-i \Delta \theta^{d}},
\end{aligned}
$$

where $\Delta \theta^{d}$ and $\phi$ are defined in (19) and (44), respectively.

The condition (80) in the normal hierarchal case requires that $\Delta \theta^{u} \simeq 0$, which implies that $\theta_{q} \simeq \Delta \theta^{d}$. We recall that $\theta_{q}$ is determined in the quark sector (realistic values of $\theta_{q}$ are between -1.3 and -1.0 as it is given in (40) ). Therefore, if we use the experimental information on $\left|V_{\mu 3}\right|$, we can relate two parameters $\phi$ and $\theta_{a}$. Below we assume that the mixing of the atmospheric neutrino is close to maximal and that $\sin \theta_{a}$ is positive, and we regard $\sin \phi$ as independent. In Fig. 6 we plot $\left|V_{e 3}\right|^{2}$ against $\sin \phi$ for different values of $R_{\nu}$ with $\theta_{q}=-1.15$. In Fig. $7, \sin \delta_{C P}$ (defined in the standard form (69) under the assumption $\sin \theta_{13}>0$ ) against $\sin \phi$ is plotted. 


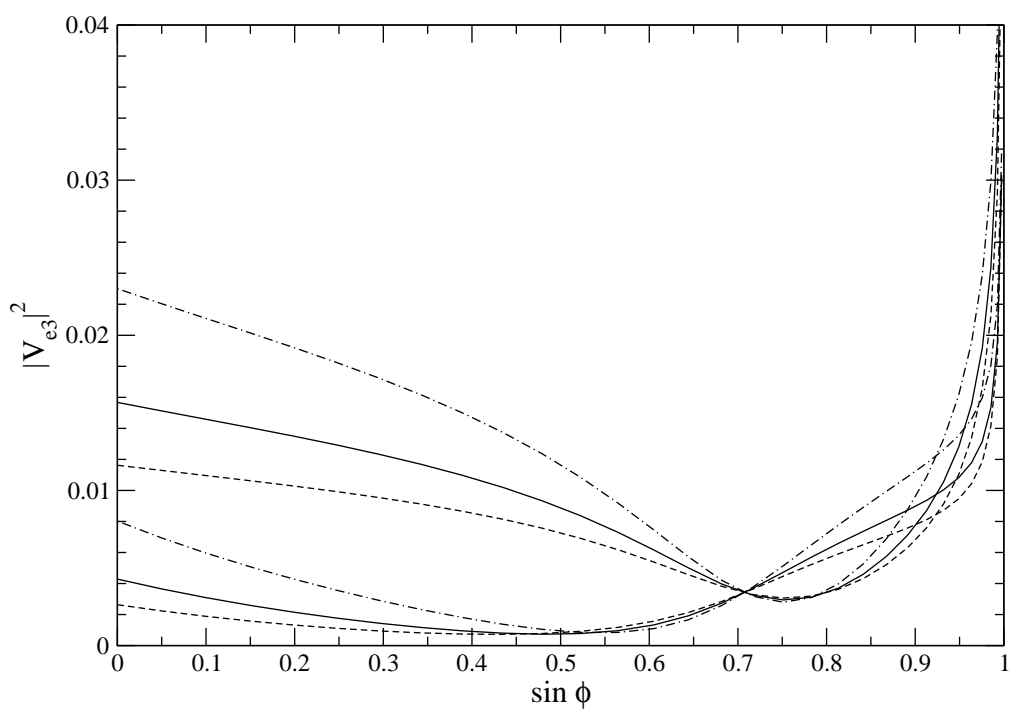

FIG. 6: $\left|V_{e 3}\right|^{2}$ against $\sin \phi$ for $R_{\nu}=0.15$ (doted), 0.19 (solid) and 0.25 (dashed-doted) with $\theta_{q}=-1.15$ and $\sin ^{2} \theta_{\nu}=0.3$, where $R_{\nu}$ and $\theta_{q}$ are defined in (83) and (27), respectively. The upper (lower) lines of each pair at $\sin \phi=0$ correspond to $\sin \theta_{\nu}\left(\simeq \sin \theta_{12}\right)=-(+) \sqrt{0.3}$. The neutrino mass spectrum is normal.

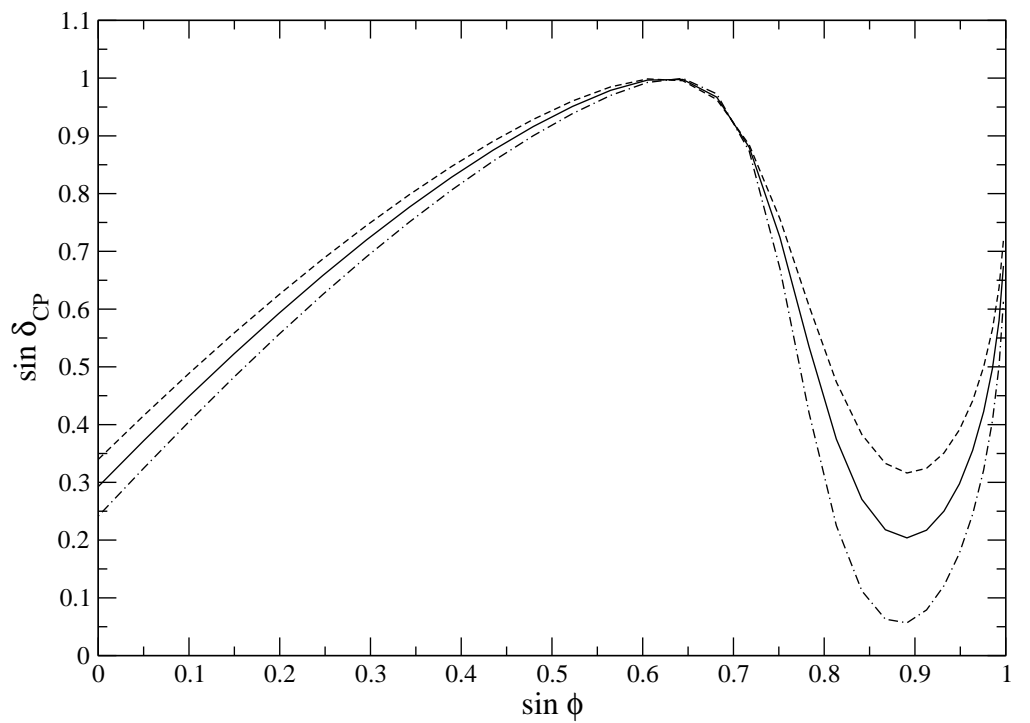

FIG. 7: $\sin \delta_{C P}$ against $\sin \phi$ for $R_{\nu}=0.15$ (doted), 0.19 (solid) and 0.25 (dashed-doted) with $\sin \theta_{\nu}=-\sqrt{0.3}$.

As we see from Figs. 6-8, in the case of a normal neutrino mass spectrum, too, precise 


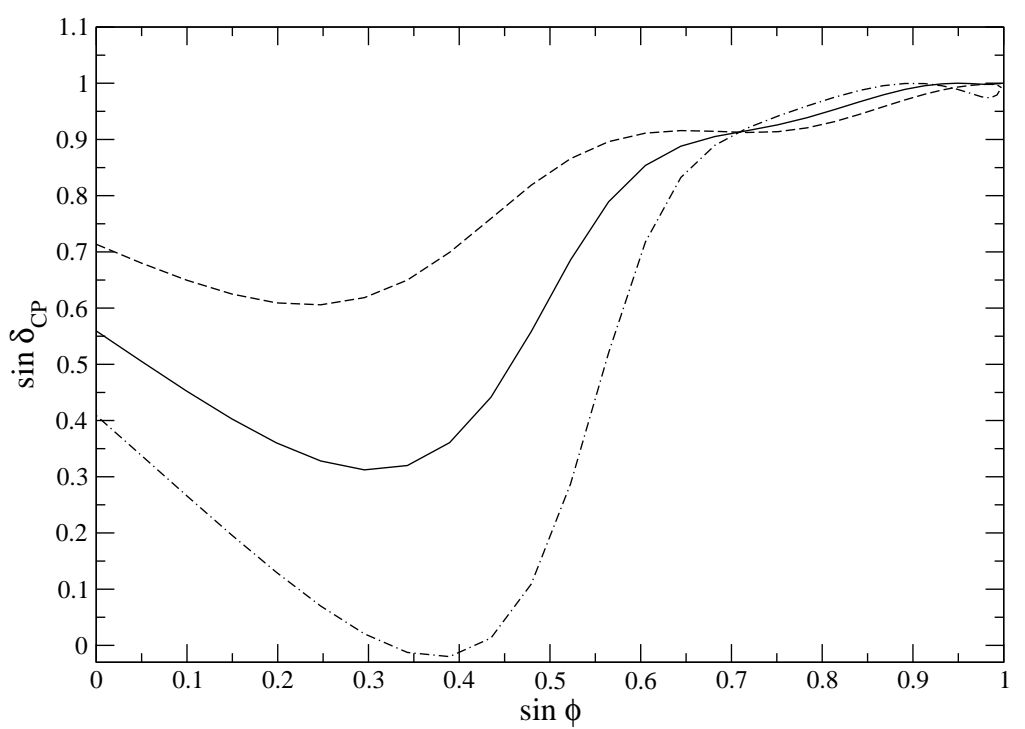

FIG. 8: The same as Fig. 7 for $\sin \theta_{\nu}=+\sqrt{0.3}$.

measurements of the physical parameters in the neutrino sector can in principle confirm the predictions of the model.

\section{E. Higgs sector}

The Higgs supermutiplets $H^{u, d}$ and $H_{3}^{u, d}$ belong to $\mathbf{2}^{\prime}$ and $\mathbf{1}^{\prime \prime \prime}$, respectively. Therefore, the singlets $H_{3}^{u, d}$ can not form a mass term. So, the $Q_{6}$ invariant superpotential is

$$
W_{H}=\mu_{H}\left(H_{1}^{u} H_{1}^{d}+H_{2}^{u} H_{2}^{d}\right)
$$

which has an accidental $U(2)$ symmetry, implying that after spontaneous symmetry breaking of the electroweak gauge symmetry, there will appear quasi Nambu-Goldstone supermultiplets. Moreover, the mass term for $H_{3}$ is absent, so that its fermionic component will remain massless even after SUSY breaking. So, they will conflict with experimental observations.

The origin of the so-called $\mu$ term is always a mysterious problem. It is usually assumed that it is related to SUSY breaking. In the present model with $Q_{6}$ family symmetry, we also worry about breaking of $Q_{6}$, because our assumption on VEVs (18) means a complete breaking of $Q_{6}$. It may be interesting to construct a Higgs sector, in which the origin of the $\mu$ term and $Q_{6}$ breaking are related to SUSY breaking. It turns out that to construct such a sector, we need to introduce a number of $S U(2)_{L}$ singlet Higgs multiplets, and also an additional abelian discrete symmetry $Z_{12 R}$, which acts as an $R$-symmetry. $Q_{6} \times Z_{12 R}$ assignments are given in 
Table 1 and 2 .

\begin{tabular}{|c|c|c|c|c|c|}
\hline & $S$ & $T$ & $X$ & $Y$ & $Z$ \\
\hline$Q_{6}$ & $\mathbf{2}$ & $\mathbf{2}^{\prime}$ & $\mathbf{1}$ & $\mathbf{1}^{\prime}$ & $\mathbf{1}^{\prime}$ \\
\hline$Z_{12 R}$ & 2 & 2 & -2 & 6 & 0 \\
\hline
\end{tabular}

Table 2. $Q_{6} \times Z_{12 R}$ assignment of the $S U(3)_{C} \times S U(2)_{L} \times U(1)_{Y}$ singlet Higgs supermultiplets.

Then we consider the following most general $Q_{6} \times Z_{12 R}$ invariant superpotential which contains the right-handed neutrino and Higgs supermultiplets:

$$
W_{H}^{\prime}=W_{I}+W_{I I}
$$

where the $Z_{12 R}$ charge of $W_{H}^{\prime}$ is $-2(\bmod 12)$, and

$$
\begin{aligned}
W_{I}= & M_{1}\left(N_{1}^{2}+N_{2}^{2}\right)+\lambda_{N} Z N_{3}^{2}+\lambda_{Z} X\left(Z^{2}-M^{2}\right), \\
W_{I I}= & \lambda_{1} X Y^{2}+\lambda_{2}\left(S_{1} S_{1}+S_{2} S_{2}\right) Y \\
& +\kappa_{1}\left(H_{1}^{u} S_{2}+H_{2}^{u} S_{1}\right) H_{3}^{d}+\kappa_{2}\left(H_{1}^{d} S_{2}+H_{2}^{d} S_{1}\right) H_{3}^{u} \\
& +\kappa_{3}\left[-\left(H_{1}^{u} H_{1}^{d}-H_{2}^{u} H_{2}^{d}\right) T_{1}+\left(H_{1}^{u} H_{2}^{d}+H_{2}^{u} H_{1}^{d}\right) T_{2}\right] .
\end{aligned}
$$

We assume that $O\left(M_{1}\right) \sim O(M)$. In the SUSY limit, there is a local minimum, where $\langle Z\rangle=M$ and all other VEVs are zero. After SUSY breaking, $X$ will acquire a VEV of order $M_{\mathrm{SUSY}}$, which will induce effective $\mu$ terms for the other Higgs supermutiplets in (91). The charges of the fields are so chosen that the most general $Q_{6} \times Z_{12 R}$ invariant Higgs superpotential (89) has an accidental parity invariance:

$$
H_{1}^{u, d} \leftrightarrow H_{2}^{u, d}, S_{1} \leftrightarrow S_{2}, T_{1} \rightarrow-T_{1},
$$

where $H_{3}^{u, d}, X, Y, Z$ and $T_{2}$ do not transform. This enables us to choose a VEV structure given by

$$
\begin{gathered}
\left\langle H_{1}^{u}\right\rangle=\left\langle H_{2}^{u}\right\rangle, \quad\left\langle H_{1}^{d}\right\rangle=\left\langle H_{2}^{d}\right\rangle,\left\langle H_{3}^{u, d}\right\rangle \neq 0, \\
\left\langle S_{1}\right\rangle=\left\langle S_{2}\right\rangle, \quad\left\langle T_{1}\right\rangle=0, \quad\left\langle T_{2}\right\rangle \neq 0 .
\end{gathered}
$$

Moreover, it is found that the superpotential $W_{H}^{\prime}$ (89) induces spontaneous CP violation, when all couplings are taken to be real. 
The Higgs sector given in this subsection may not be minimal. It is however clear that the Higgs sector is related to SUSY breaking, so that there will be different modifications. The important point is that the effective superpotential for the $S U(2)_{L}$ doublet Higgs multiplets should possess the $H_{1} \leftrightarrow H_{2}$ symmetry to ensure the VEV structure (18). Therefore, instead of introducing the $S, T, \ldots$ singlets fields, one may assume that $Q_{6}$ is explicitly, but softly broken by dimension 3 terms. If we demand that these soft terms do not break the $H_{1} \leftrightarrow H_{2}$ symmetry, it must be of the form

$$
\begin{aligned}
W_{\text {eff }}= & \mu_{1}\left(H_{1}^{u} H_{1}^{d}+H_{2}^{u} H_{2}^{d}\right)+\mu_{3} H_{3}^{u} H_{3}^{d}+\mu_{13}\left(H_{1}^{u}+H_{2}^{u}\right) H_{3}^{d} \\
& +\mu_{31} H_{3}^{u}\left(H_{1}^{d}+H_{2}^{d}\right)+\mu_{12}\left(H_{1}^{u} H_{2}^{d}+H_{2}^{u} H_{1}^{d}\right) .
\end{aligned}
$$

Since the $\mu$ parameter results from VEVs, they may be complex.

In models with more than one Higgs $S U(2)_{L}$ doublet, as in the case of the present model, tree-level FCNCs exist in the Higgs sector. They contribute, for instance, to the mass difference $\Delta m_{K}$ of $K^{0}$ and $\bar{K}^{0}$, which will give severe constraints on the model, especially on the heavy Higgs masses. [See for instance [25, 58].] This is one of the important questions, but detailed investigation of this question is beyond the scope of the present paper. So we would like to leave this problem to future work.

\section{DISCUSSIONS}

Here we would like to discuss further features of the $Q_{6}$ model.

\section{A. Anomalies of discrete symmetries}

Quantum gravitational effects violate all global symmetries, while they respect all local symmetries [59]. When a local gauge group is spontaneously broken, a certain discrete subgroup may survive [60], which is respected by gravity. A necessary condition for a discrete symmetry group to be a subgroup of a gauge group is the absence of anomalies [45, 46, 47].

We start with $Q_{6}$ anomalies e, $Q_{6}\left[S U(2)_{L}\right]^{2}$ and $Q_{6}\left[S U(3)_{C}\right]^{2}$. Since the anomaly in $Q_{6}\left[U(1)_{Y}\right]^{2}$ depends on the normalization of $U(1)_{Y}$, it does give a useful information. Similarly, heavy fields can contribute to the $\left[Q_{6}\right]^{3}$ anomaly so that it does not constrain the low-energy

\footnotetext{
${ }^{\mathrm{e}} Q_{6}$ anomalies are also considered in $[18]$.
} 
spectrum. To compute anomalies, we note that $A_{Q_{6}},\left(A_{Q_{6}}\right)^{2}, \ldots,\left(A_{Q_{6}}\right)^{5}$ correspond to $Z_{6}$ rotations, and $B_{Q_{6}}$ to $Z_{4}$ rotations. $\left[A_{Q_{6}}\right.$ and $B_{Q_{6}}$ are defined in (5) and (6).] One can easily convince oneself that the doublets of $Q_{6}$ can not contribute to the anomalies, because they always contain two components, one with a plus charge and the other one with a negative charge in the same amount. The charges of $Q_{6}$ singlets under $\left(Z_{6}, Z_{4}\right)$ are given by

$$
\mathbf{1}:(0,0), \mathbf{1}^{\prime}:(0,2), \mathbf{1}^{\prime \prime}:(3,-1), \mathbf{1}^{\prime \prime \prime}:(3,1)
$$

From Table 1 we can read off the corresponding charges of the matter supermultiplets, and we then compute the anomaly coefficients:

$$
\begin{aligned}
& Z_{6}\left[S U(2)_{L}\right]^{2}: 2 A_{2}=3(\bmod 6), Z_{6}\left[S U(3)_{C}\right]^{2}: 2 A_{3}=3(\bmod 6) \\
& Z_{4}\left[S U(2)_{L}\right]^{2}: 2 A_{2}=2(\bmod 4), Z_{4}\left[S U(3)_{C}\right]^{2}: 2 A_{3}=2(\bmod 4)
\end{aligned}
$$

$Z_{12 R}\left[S U(2)_{L}\right]^{2}$ and $Z_{12 R}\left[S U(3)_{C}\right]^{2}$ anomalies can also be straightforwardly computed, and we find

$$
Z_{12 R}\left[S U(2)_{L}\right]^{2}: 2 A_{2}=6(\bmod 12), Z_{12 R}\left[S U(3)_{C}\right]^{2}: 2 A_{3}=6(\bmod 12)
$$

Anomalies of a discrete symmetry can be cancelled by the Green-Schwarz mechanism, if

$$
\frac{A_{2}+m N / 2}{k_{2}}=\frac{A_{3}+m^{\prime} N / 2}{k_{3}}
$$

is satisfied, where $k$ 's ( the Kac-Moody levels ) and $m, m^{\prime}$ are integers, and $N$ stands for the order of $Z_{N}$ [45, 46, 47]. Therefore, all the anomalies cancel if we choose

$$
k_{2}=k_{3},
$$

for instance.

\section{B. $R$-parity and Baryon number violating operators}

Just because of $Q_{6}$ symmetry, it turns out that $R$-parity violating couplings are almost absent. Out of the $96 R$-parity breaking cubic couplings that are allowed in the MSSM superpotential, $Q_{6}$ allows only one coupling

$$
\lambda^{\prime}\left[\left(L_{1} Q_{2}+L_{2} Q_{1}\right) D_{1}^{c}+\left(L_{1} Q_{1}-L_{2} Q_{2}\right) D_{2}^{c}\right]
$$


Many couplings vanish because of color antisymmetry and $S U(2)_{L}$ antisymmetry. Furthermore, all baryon number violating cubic terms are forbidden by $Q_{6}$ alone. This means that there is no proton decay problem in the present model ${ }^{\mathrm{f}}$. The natural value of $\lambda^{\prime}$ is about $10^{-3}$, consistent with Yukawa couplings of charged sector. This term will induce a neutrino mass proportional to the strange quark mass at one loop. The magnitude may be of order $10^{-2} \mathrm{eV}$.

\section{The SUSY flavor problem and phase alignment}

Since $Q_{6}$ is an intact symmetry at $M_{\mathrm{SUSY}}$ (except for the mass term of $N_{3}^{c}$, which results from the $\mathrm{VEV}$ of $Z$ in (90)), it is natural to assume that the SSB sectors also respect $Q_{6}$ symmetry. From the results of [40, 41], in which a detailed analysis on FCNCs and CP violations in a supersymmetric extension of the SM with an nonabelian discrete flavor symmetry $S_{3}$ has been made, we may expect that $Q_{6}$ suppresses strongly FCNC and CP violating processes that are induced by the SSB terms. However, the constraints coming from the EDM of neutron, electron and mercury atom [42, 51, 52, 53] are very severe. Recent experiment on mercury atom [50] requires that the imaginary part of the dimensionless quantity $\left(\delta_{11}^{d}\right)_{L R}$ has to satisfy $\left|\operatorname{Im}\left(\delta_{11}^{d}\right)_{L R}\right|<6.7 \times 10^{-8}\left(\tilde{m}_{q} / 100 \mathrm{GeV}\right)^{2}[51]$, where $\tilde{m}_{q}$ is the average of the squark masses. Similar constraints exist for $\left(\delta_{11}^{u}\right)_{L R}$ and $\left(\delta_{11}^{e}\right)_{L R}$, too. The quantity $\delta_{L R}^{d}$ is defined as [42]

$$
\delta_{L R}^{d}=\frac{U_{d}^{T} \tilde{\mathbf{m}}_{d L R}^{2} U_{d^{c}}}{\tilde{m}_{q}^{2}},
$$

where $\tilde{\mathbf{m}}_{d L R}^{2}$ resulting from the A terms is the so-called left-right mass matrix for the bosonic components of $D$ and $D^{c}$. The unitary matrices are those that rotate the fermionic superpartners, i.e. $U_{d}=P_{d} O_{d}, U_{d^{c}}=P_{d^{c}} O_{d^{c}}$, where the phase matrices $P_{d}$ and $P_{d^{c}}$ are defined in in (22) and (23), respectively. Since we assume that the CP phases are spontaneously induced, and thanks to $Q_{6}$ symmetry the mass matrix $\tilde{\mathbf{m}}_{d L R}^{2}$ has exactly the same structure as the mass matrix (17), we conclude that $\left(\delta_{11}^{d}\right)_{L R}$ is a real number. From the same reason, all $\delta_{L R}$ are real. Thus, we can satisfy the most stringent constraint on the A terms without any fine tuning. This is true not only at a particular energy scale, but also for the entire energy scale, which should be compared with the case of the MSSM [52].

\section{Acknowledgments}

\footnotetext{
${ }^{\mathrm{f}}$ Anomaly-free abelian discrete symmetries to suppress proton decay have been considered in [47].
} 
This work is supported by the Grants-in-Aid for Scientific Research from the Japan Society for the Promotion of Science (No. 13135210).

\section{APPENDIX A: MULTIPLICATION RULES}

Here we give the multiplication rules for $D_{3}, D_{4}, D_{6}, D_{8}, Q_{4}$ and $Q_{8}$. The multiplication rules (without the Clebsch-Gordan coefficients) for finite groups with larger orders are given in [44].

1. $D_{3}\left(S_{3}\right)$

The irreps of $D_{3}$ are $\mathbf{2}, \mathbf{1}, \mathbf{1}^{\prime}$.

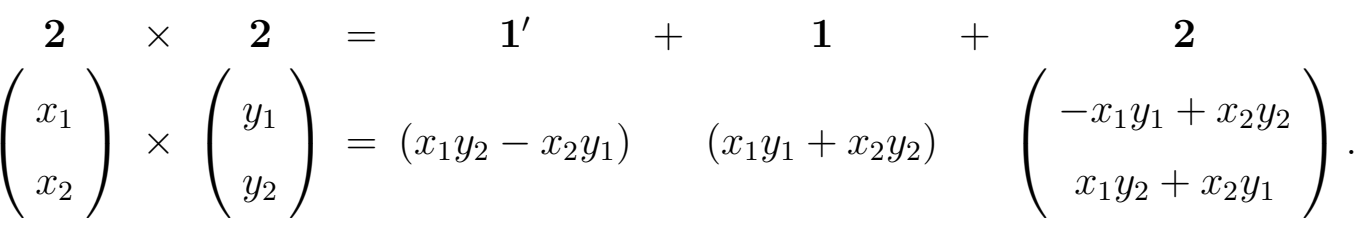

2. $D_{4}$

The irreps of $D_{4}$ are $\mathbf{2}, \mathbf{1}, \mathbf{1}^{\prime}, \mathbf{1}^{\prime \prime}, \mathbf{1}^{\prime \prime \prime}$.

$$
\begin{aligned}
& 2 \times 2=1 \quad+\quad 1^{\prime \prime}
\end{aligned}
$$

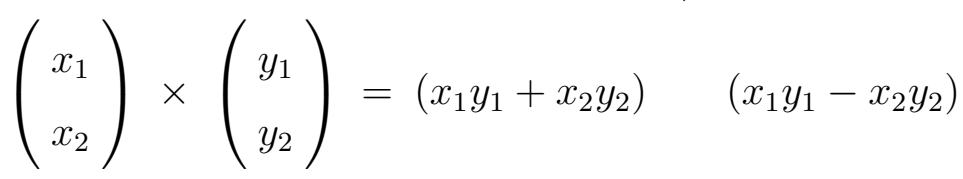

$$
\begin{aligned}
& +\mathbf{1}^{\prime} \quad+\quad \mathbf{1}^{\prime \prime \prime} \\
& \left(x_{1} y_{2}-x_{2} y_{1}\right) \quad\left(x_{1} y_{2}+x_{2} y_{1}\right) \text {. }
\end{aligned}
$$

\section{3. $D_{6}$}

The irreps of $D_{6}$ are $\mathbf{2}, \mathbf{2}^{\prime}, \mathbf{1}, \mathbf{1}^{\prime}, \mathbf{1}^{\prime \prime}, \mathbf{1}^{\prime \prime \prime}$.

$$
\begin{aligned}
& 2 \times 2=1^{\prime} \quad+\quad \begin{array}{llll}
2^{\prime} & +
\end{array}
\end{aligned}
$$

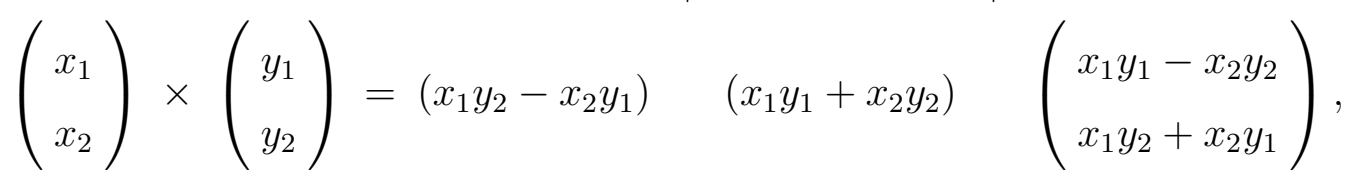




$$
\begin{aligned}
& \mathbf{2}^{\prime} \times \mathbf{2}^{\prime}=\begin{array}{llllll} 
& \mathbf{1} & + & \mathbf{1}^{\prime} & + & 2^{\prime}
\end{array}
\end{aligned}
$$

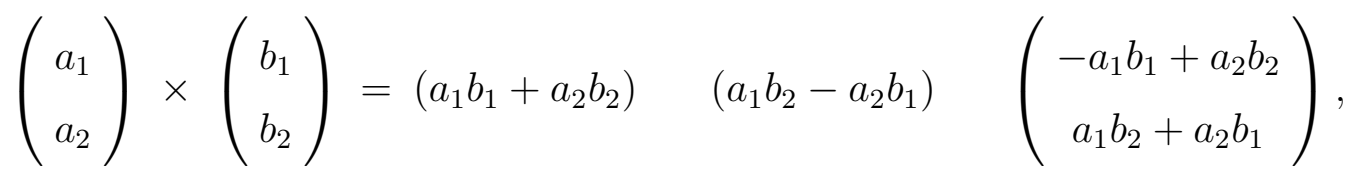

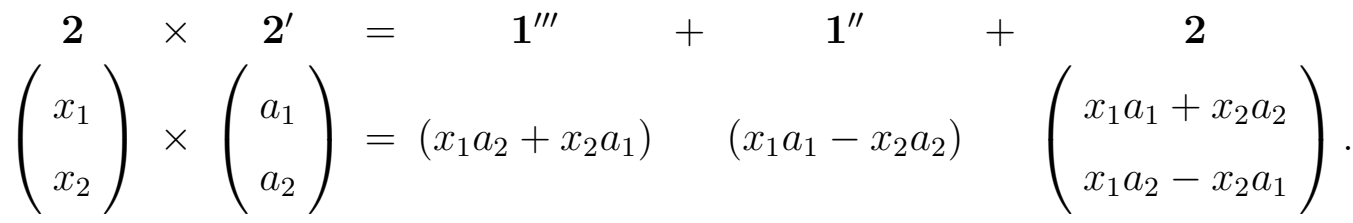

4. $D_{8}$

The irreps of $D_{8}$ are $\mathbf{2}, \mathbf{2}^{\prime}, \mathbf{2}^{\prime \prime}, \mathbf{1}, \mathbf{1}^{\prime}, \mathbf{1}^{\prime \prime}, \mathbf{1}^{\prime \prime \prime}$.

$$
\begin{aligned}
& 2 \times 2=1^{\prime}+x^{\prime}+2^{\prime}
\end{aligned}
$$

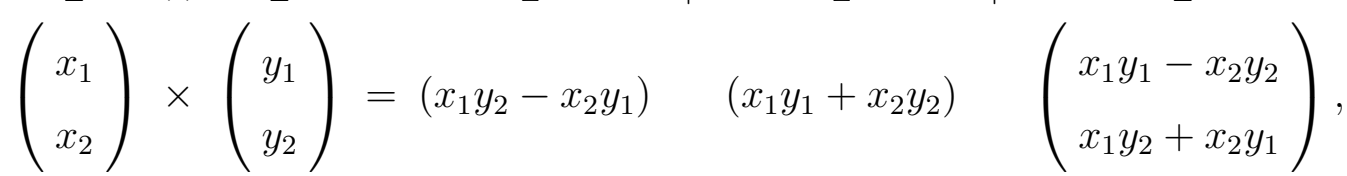

$$
\begin{aligned}
& 2^{\prime} \times 2^{\prime}=1+1^{\prime \prime} \\
& \left(\begin{array}{l}
a_{1} \\
a_{2}
\end{array}\right) \times\left(\begin{array}{l}
b_{1} \\
b_{2}
\end{array}\right)=\left(a_{1} b_{1}+a_{2} b_{2}\right) \quad\left(a_{1} b_{1}-a_{2} b_{2}\right) \\
& +\mathbf{1}^{\prime}+\mathbf{1}^{\prime \prime \prime} \\
& \left(a_{1} b_{2}-a_{2} b_{1}\right) \quad\left(a_{1} b_{2}+a_{2} b_{1}\right)
\end{aligned}
$$

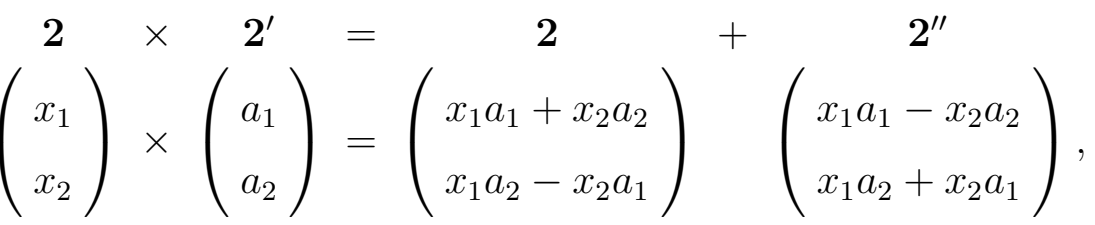

$$
\begin{aligned}
& \begin{array}{c}
2^{\prime \prime} \times \mathbf{2}^{\prime}= \\
\left(\begin{array}{c}
t_{1} \\
t_{2}
\end{array}\right) \times\left(\begin{array}{c}
a_{1} \\
a_{2}
\end{array}\right)=\left(\begin{array}{c}
-t_{1} a_{1}-t_{2} a_{2} \\
t_{1} a_{2}-t_{2} a_{1}
\end{array}\right){ }^{\prime \prime} \quad\left(\begin{array}{c}
t_{1} a_{1}-t_{2} a_{2} \\
-t_{1} a_{2}-t_{2} a_{1}
\end{array}\right) .
\end{array}
\end{aligned}
$$




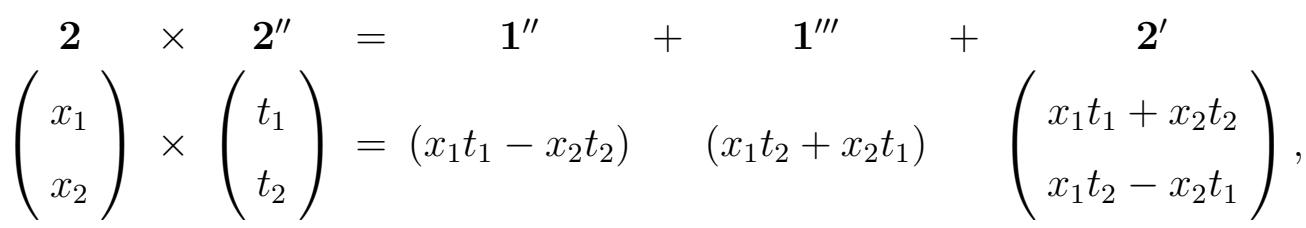

$$
\begin{aligned}
& 2^{\prime \prime} \times 2^{\prime \prime}=\mathbf{1}^{\prime} \quad \mathbf{1}^{\prime}+\mathbf{2}^{\prime}
\end{aligned}
$$

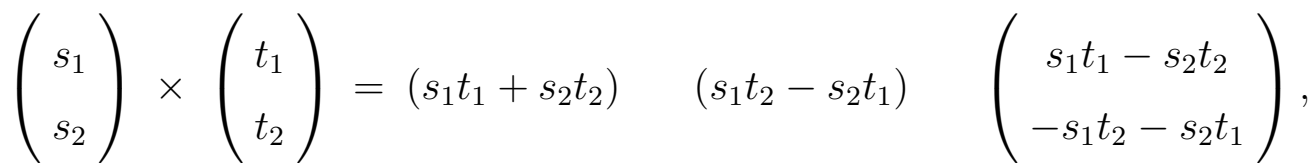

5. $Q_{4}$

The irreps of $Q_{4}$ are $\mathbf{2}, \mathbf{1}, \mathbf{1}^{\prime}, \mathbf{1}^{\prime \prime}, \mathbf{1}^{\prime \prime \prime}$, where $\mathbf{2}$ is pseudo-real, and the singlets are real.

$$
\begin{aligned}
& 2 \times 2=1^{\prime}+1^{\prime \prime \prime}
\end{aligned}
$$

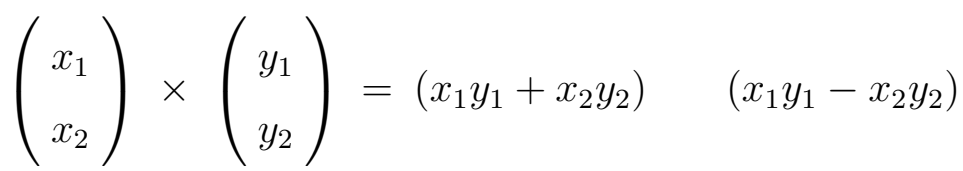

$$
\begin{aligned}
& +\quad 1+1^{\prime \prime} \\
& \left(x_{1} y_{2}-x_{2} y_{1}\right) \quad\left(x_{1} y_{2}+x_{2} y_{1}\right),
\end{aligned}
$$

6. $Q_{6}$

The multiplication rules for $Q_{6}$ are given in Sect. II.

7. $Q_{8}$

The irreps of $Q_{8}$ are $\mathbf{2}, \mathbf{2}^{\prime}, \mathbf{2}^{\prime \prime}, \mathbf{1}, \mathbf{1}^{\prime}, \mathbf{1}^{\prime \prime}, \mathbf{1}^{\prime \prime \prime}$. All singlets and $\mathbf{2}^{\prime}$ are real representations.

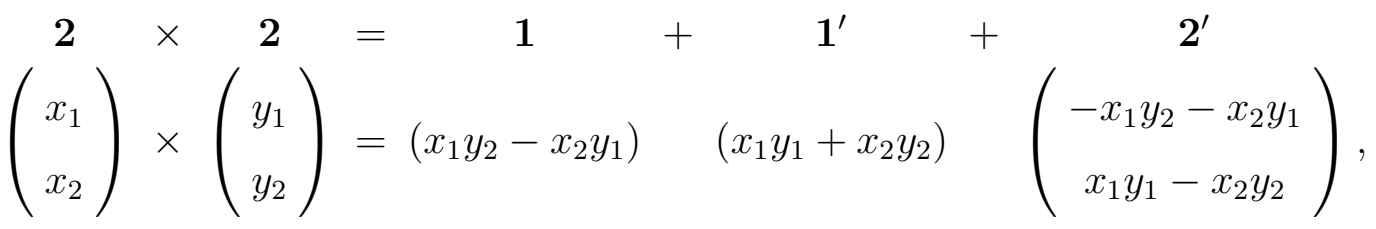




$$
\begin{aligned}
& 2^{\prime} \times 2^{\prime}=1 \quad+1^{\prime \prime} \\
& \left(\begin{array}{l}
a_{1} \\
a_{2}
\end{array}\right) \times\left(\begin{array}{l}
b_{1} \\
b_{2}
\end{array}\right)=\left(a_{1} b_{1}+a_{2} b_{2}\right) \quad\left(a_{1} b_{1}-a_{2} b_{2}\right) \\
& +\quad \mathbf{1}^{\prime} \quad+\quad \mathbf{1}^{\prime \prime \prime} \\
& \left(a_{1} b_{2}-a_{2} b_{1}\right) \quad\left(a_{1} b_{2}+a_{2} b_{1}\right)
\end{aligned}
$$

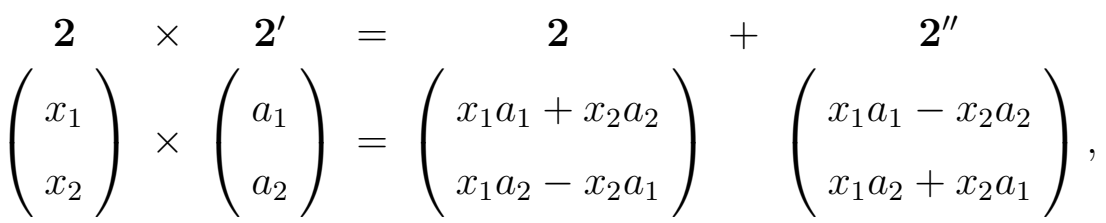

$$
\begin{aligned}
& \begin{array}{c}
2^{\prime \prime} \times \mathbf{2}^{\prime}= \\
\left(\begin{array}{c}
t_{1} \\
t_{2}
\end{array}\right) \times\left(\begin{array}{c}
a_{1} \\
a_{2}
\end{array}\right)=\left(\begin{array}{c}
-t_{1} a_{1}-t_{2} a_{2} \\
t_{1} a_{2}-t_{2} a_{1}
\end{array}\right){ }^{\prime \prime} \quad\left(\begin{array}{c}
t_{1} a_{1}-t_{2} a_{2} \\
-t_{1} a_{2}-t_{2} a_{1}
\end{array}\right),
\end{array} \\
& 2 \times 2^{\prime \prime}=1^{\prime \prime \prime}+1^{\prime \prime}+2^{\prime}
\end{aligned}
$$

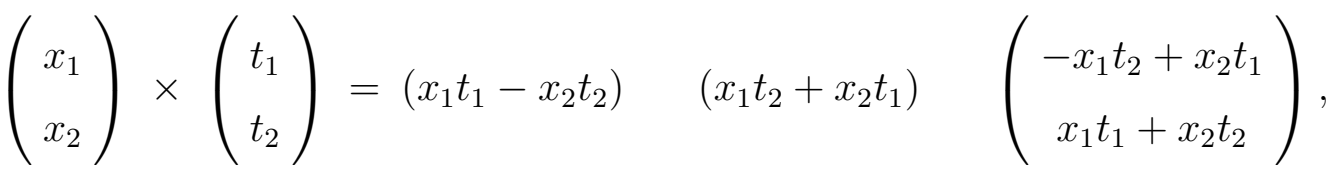

$$
\begin{aligned}
& 2^{\prime \prime} \times 2^{\prime \prime}=1^{\prime} \quad+\quad 1 \quad+2^{\prime}
\end{aligned}
$$

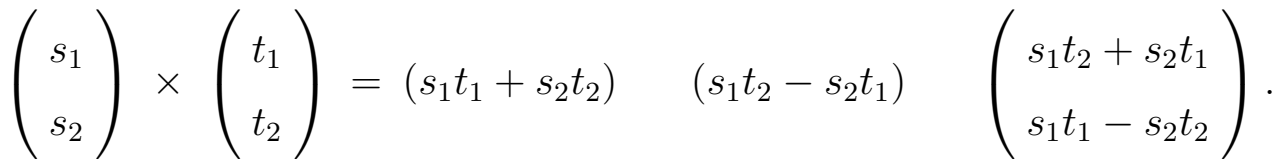

[1] For recent reviews, see, for instance, S.P. Martin, hep-ph/9709356 and references therein.

[2] S. Weinberg, in Transactions of the New York Academy of Sciences, Series II, Vol. 38, 185 (1977).

[3] F. Wilczek and A. Zee, Phys. Rev. Lett. 42, 421 (1979).

[4] H. Fritzsch, Phys. Lett. B 73, 317 (1978); Nucl. Phys. B155, 189 (1979).

[5] H. Fritzsch and Z.Z. Xing, Prog. Part. Nucl. Phys. 45, 1 (2000).

[6] G.C. Branco, L. Lavoura and F. Mota, Phys. Rev. D39, 3443 (1989).

[7] S. Dimopoulos, L.J. Hall and S. Raby, Phys. Rev. Lett. 68, 1984 (1992); Phys. Rev. D45, 4192 (1992).

[8] A.J. Davies and X-G. He, Phys .Rev. D46, 3208 (1992). 
[9] K.S. Babu and Q. Shafi, Phys. Lett. B311,172 (1993); Phys. Rev. D47, 5004 (1993).

[10] Y. Achiman, Phys. Lett. B329, 33 (1994).

[11] B. Dutta and S. Nandi, Phys. Lett. B366, 281 (1996).

[12] K. Harayama, N. Okamura, A.I. Sanda and Z-Z. Xing, Prog. Theor. Phys. 97, 781 (1997).

[13] Y. Koide, Mod. Phys. Lett. A12, 2655 (1997).

[14] D. Dooling and K. Kang, Phys. Lett. B455, 264 (1999).

[15] Z-Z. Xing, Phys. Lett.B550, 178 (2002).

[16] M. Fukugita, M. Tanimoto and T. Yanagida, Phys. Lett. B562, 273 (2003).

[17] J.C. Pati and A. Salam, Phys. Rev. Lett. 31, 661 (1973).

[18] P.H. Frampton and T.W. Kephart, Int. J. Mod. Phys. A10, 4689 (1995).

[19] S. Pakvasa and H. Sugawara, Phys. Lett. B73, 61 (1978) ; Phys. Lett. B82,105 (1979).

[20] H. Harari, H. Haut and T. Weyers, Phys. Lett. B78, 459 (1978).

[21] E. Derman, Phys. Rev. D19, 317 (1979); E. Derman and H.-S. Tsao, Phys. Rev. D20, 1207 (1979).

[22] D. Wyler, Phys. Rev. D19, 3369 (1979).

[23] G. Segrè and H.A. Weldon, Phys. Rev. Lett. 42, 1191 (1979).

[24] H. Sato, Nucl. Phys. B148, 433 (1979).

[25] Y. Yamanaka, H. Sugawara and S. Pakvasa , Phys. Rev. D25, 1895 (1982); Erratum D29, 2135 (1984).

[26] Y. Koide, Lett. Nuovo Cimento 34, 201 (1982); Phys. Lett. B120, 161 (1983); Phys. Rev. D28, $252(1983)$.

[27] E. Ma and G. Rajasekaran, Phys. Rev. D64, 113012 (2001); E. Ma, Mod. Phys. Lett. A17, 627 (2002); 2361 (2002).

[28] K.S. Babu, E. Ma and J.W.F. Valle, Phys. Lett. B552, 207 (2003).

[29] E. Ma, Phys .Rev. D70, 031901 (2004).

[30] E. Ma, Phys. Rev. D43, R2761 (1991).

[31] J. Kubo, A. Mondragón, M. Mondragón and E. Rodríguez-Jáuregui, Prog. Theor. Phys. 109, 795 (2003).

[32] J. Kubo, Phys. Lett. B578, 156 (2004).

[33] S.-L. Chen, M. Frigerio and E. Ma, hep-ph/0404084

[34] W. Grimus and L. Lavoura, Phys. Lett. B572, 189 (2003).

[35] W. Grimus, A.S. Joshipura, S. Kaneko, L. Lavoura and M. Tanimoto, JHEP 0407, 078 (2004).

[36] M. Frigerio, S. Kaneko, E. Ma and M. Tanimoto, hep-ph/0409187

[37] L.J. Hall and H. Murayama, Phys. Rev. Lett. 75, 3985 (1995); C.D. Carone, L.J. Hall and H. Murayama, Phys. Rev. D53, 6282 (1996).

[38] K. Hamaguchi, M. Kakizaki and M. Yamaguchi, Phys. Rev. D68, 056007 (2003).

[39] K.S. Babu, T. Kobayashi and J. Kubo, Phys. Rev. D67, 075018 (2003).

[40] T. Kobayashi, J. Kubo and H. Terao, Phys. Lett. B568, 83 (2003).

[41] Ki-Y. Choi, Y. Kajiyama, J. Kubo and H. M. Lee, hep-ph/0402026, to published in Phys. Rev. D.

[42] F. Gabbiani, E. Gabrielli, A. Masiero and L. Silvestrini, Nucl. Phys. B477, 321 (1996).

[43] J. Ellis, S. Ferrara and D.V. Nanopoulos, Phys. Lett. B114, 231 (1982); W. Buchmüller and D. Wyler, Phys. Lett. B121, 321 (1983); J. Polchinski and M.B. Wise, Phys. Lett. B125, 393 (1983); F. del Aguila, M.B. Gavela, J.A. Grifols and A. Méndez, Phys. Lett. B126, 71 (1983); D.V. Nanopoulos and M. Srednicki, Phys. Lett. B128, 61 (1983). 
[44] P.H. Frampton and T.W. Kephart, Phys. Rev. D64, 086007 (2001).

[45] T. Banks and M. Dine, Phys. Rev. D45, 1424 (1992).

[46] L.E. Ibáñez and G.G. Ross, Phys. Lett. B260, 291 (1991); L.E. Ibáñez and G.G. Ross, Nucl. Phys. B368, 3 (1992); L.E. Ibáñez, Nucl. Phys. B398, 301 (1993).

[47] K.S. Babu, I. Gogoladze and K. Wang, Phys. Lett. B560, 214 (2003); Nucl. Phys. B660, 322 (2003).

[48] E.D. Commins et al., Phys. Rev. A50, 2960 (1994).

[49] P.G. Harris et al., Phys. Rev. Lett. 82, 904 (1999).

[50] M.V. Romalis et al., Phys. Rev. Lett. 86, 2505 (2001).

[51] S. Abel, S. Khalil and O. Lebedev, Nucl. Phys. B606, 151 (2001).

[52] K.S. Babu, and T. Enkhbat, hep-ph/0406003.

[53] Particle Data Group, Phys. Lett . B592, 1 (2004).

[54] H.D. Kim, S. Raby and L. Schradin, hep-ph/0401169.

[55] M. Maltoni, T. Schwetz, M.A. Tortola, J.W.F. Valle, hep-ph/0405172.

[56] D.N. Spergel et al., Astrophys. J. Suppl. 148, 175 (2003).

[57] H.V. Klapdor-Kleingrothaus et al., Eur. Phys. J. A12, (2001) 147; C.E. Aalseth et al., Phys. Atm. Nucl. 63, 1268 (2000); H.V. Klapdor-Kleingrothaus, A. Dietz, H.L. Harney and I.V. Krivosheina, Mod. Phys. Lett. A 16, 2409 (2001).

[58] T. Brown, N. Desphande, S. Pakvasa and H. Sugawara, Phys. Lett. B141, 95 (1984); N.G. Deshpande, X-G. He, Phys. Rev. D49, 4812 (1994).

[59] S.W. Hawking, Phys. Lett. B195, 337 (1987); G.V. Lavrelashvili, V.A. Rubakov and P.G. Tinyakov, JETP Lett. 46, 167 (1987); S. Giddings and A. Strominger, Nucl. Phys. B306, 349 (1988); Nucl. Phys. B321, 481 (1989); L.F. Abbot and M. Wise, Nucl. Phys. B325, 687 (1989); S. Coleman and K. Lee, Nucl. Phys. B329, 389 (1989).

[60] L. Krauss and F. Wilczek, Phys. Rev. Lett. 62, 1221 (1989). 